\title{
Prostaglandin signaling suppresses beneficial microglial function in Alzheimer's disease models
}

\author{
Jenny U. Johansson, ${ }^{1}$ Nathaniel S. Woodling ${ }^{1,2}$ Qian Wang, ${ }^{1}$ Maharshi Panchal, ${ }^{1}$ Xibin Liang, ${ }^{1}$ Angel Trueba-Saiz, ${ }^{3}$ \\ Holden D. Brown, ${ }^{1}$ Siddhita D. Mhatre, ${ }^{1}$ Taylor Loui, ${ }^{1}$ and Katrin I. Andreasson ${ }^{1}$ \\ 'Department of Neurology and Neurological Sciences, Stanford University School of Medicine, Stanford, California, USA. ${ }^{2}$ Neurosciences Graduate Program, Stanford University, Stanford, California, USA. \\ ${ }^{3}$ Functional and Systems Neurobiology Department, Cajal Institute, CSIC, Madrid, Spain.
}

\begin{abstract}
Microglia, the innate immune cells of the CNS, perform critical inflammatory and noninflammatory functions that maintain normal neural function. For example, microglia clear misfolded proteins, elaborate trophic factors, and regulate and terminate toxic inflammation. In Alzheimer's disease (AD), however, beneficial microglial functions become impaired, accelerating synaptic and neuronal loss. Better understanding of the molecular mechanisms that contribute to microglial dysfunction is an important objective for identifying potential strategies to delay progression to AD. The inflammatory cyclooxygenase/ prostaglandin E2 ( $C O X / \mathrm{PCE}_{2}$ ) pathway has been implicated in preclinical AD development, both in human epidemiology studies and in transgenic rodent models of AD. Here, we evaluated murine models that recapitulate microglial responses to $A \beta$ peptides and determined that microglia-specific deletion of the gene encoding the $\mathrm{PCE}_{2}$ receptor EP2 restores microglial chemotaxis and $A \beta$ clearance, suppresses toxic inflammation, increases cytoprotective insulin-like growth factor 1 (IGF1) signaling, and prevents synaptic injury and memory deficits. Our findings indicate that EP2 signaling suppresses beneficial microglia functions that falter during AD development and suggest that inhibition of the COX/PGE $/$ /EP2 immune pathway has potential as a strategy to restore healthy microglial function and prevent progression to $A D$.
\end{abstract}

\section{Introduction}

Alzheimer's disease (AD), a neurodegenerative disorder associated with protein misfolding and aggregation in the brain, is the most common memory disorder, and its prevalence is expected to triple by the year 2050 (1). The widely considered "amyloid hypothesis" of $A D$ causation posits that accumulation of amyloid $\beta_{42}\left(A \beta_{42}\right)$ triggers inflammation, tau hyperphosphorylation, and synaptic and neuronal loss, leading to cognitive decline $(2,3)$. Recent studies, however, indicate that brain $\mathrm{A} \beta_{42}$ accumulates in subjects that do not exhibit dementia, which suggests that $A \beta_{42}$ accumulation may be necessary but not sufficient for development of cognitive impairment (4) and that additional factors are required to tip the balance toward progression to AD dementia.

Recent genetic studies of late-onset $\mathrm{AD}$ have identified ADassociated genes that are involved in the innate immune response and are expressed in microglia, the resident myeloid cells of the CNS. Microglial genes associated with AD include CD33 (5-7), TREM2 $(8,9)$, and CR1 $(10,11)$; together with additional studies (12), these findings are indicative of an important role of microglia in maintaining local brain homeostasis and preventing $\mathrm{A} \beta_{42}$-mediated synaptic and inflammatory injury. Notably, clearance of accumulating $A \beta_{42}$ is dependent on effective sensing by microglia (mediated by chemokines), followed by $\mathrm{A} \beta_{42}$ degradation. Moreover, prolonged exposure to proinflammatory cytokines or accumulating $\mathrm{A} \beta_{42}$ peptides cause microglia to lose their normal abilities to clear

Conflict of interest: The authors have declared that no conflict of interest exists. Submitted: June 10, 2014; Accepted: October 30, 2014.

Reference information: / Clin Invest. 2015;125(1):350-364. doi:10.1172/JCI77487. toxic proteins and control inflammation $(13,14)$, a detrimental phenotype in the context of age-associated $\mathrm{A} \beta_{42}$ accumulation. Thus, microglia are emerging as critical regulators of innate immune responses in AD and, more broadly, in other neurodegenerative disorders, and understanding the molecular and cellular mechanisms that cause microglial dysfunction may help identify strategies to restore healthy microglial function and prevent development of AD.

A longstanding observation in epidemiological studies of normal aging populations has been that NSAIDs, which inhibit cyclooxygenase-1 (COX-1) and COX-2 and prostaglandin (PG) production, prevent development of $\mathrm{AD}$ (15-18). In addition, early-stage $\mathrm{AD}$ is characterized by increased cerebrospinal fluid levels of $\operatorname{PGE}_{2}(19,20)$, supporting the hypothesis that inflammatory actions of brain $\mathrm{COX} / \mathrm{PGE}_{2}$ may underlie preclinical development of $\mathrm{AD}$. Consistently, studies in $\mathrm{AD}$ model mice demonstrate reduced amyloid pathology with global deletion of individual $\mathrm{PGE}_{2} \mathrm{G}$ protein-coupled receptors (21-23), and additional studies have shown a suppressive signaling effect of the $\mathrm{PGE}_{2}$ receptor EP2 on $\mathrm{A} \beta_{42}$ phagocytosis $(24,25)$. These studies, along with the recent demonstration of a broad regulatory function of EP2 signaling on cell cycle, cytoskeletal, and immune genes in quiescent microglia (26), suggest that microglial EP2 signaling may be a general suppressor of immune and nonimmune processes that protect against onset and progression of $\mathrm{AD}$ pathology. To investigate this hypothesis, we used in vitro and in vivo mouse models that recapitulate acute and chronic aspects of microglial responses to $A \beta$ peptides. Our findings demonstrate that microglial EP2 signaling suppresses multiple processes critical to microglial maintenance of homeostasis in vivo, notably 
A

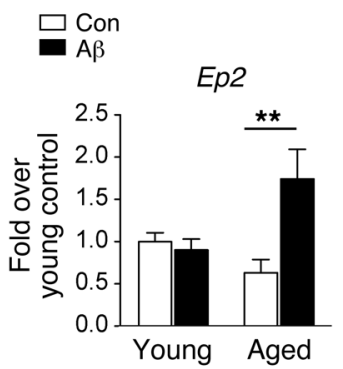

C

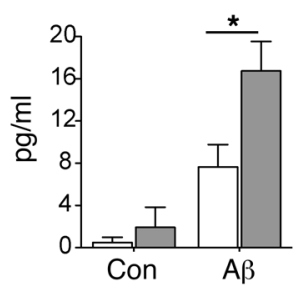

B

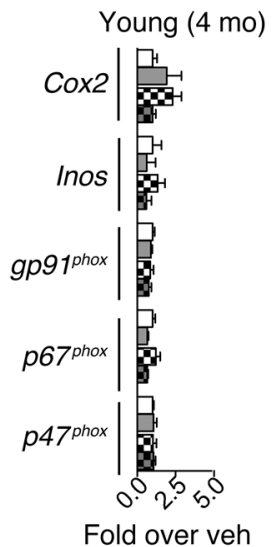

D

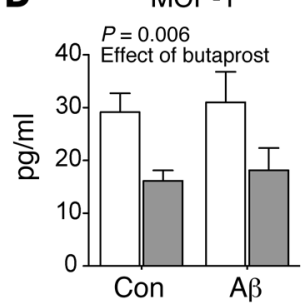

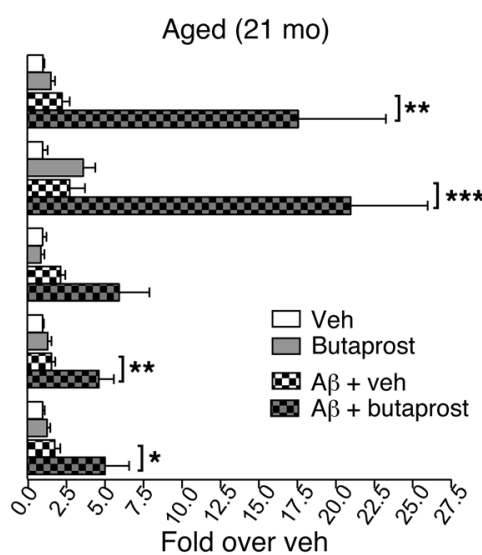

MIP-1 $\alpha$

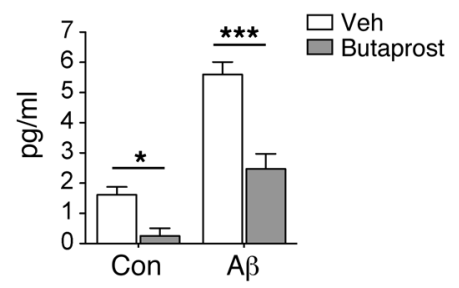

E

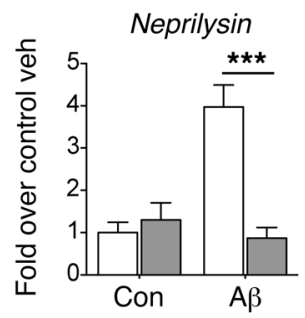

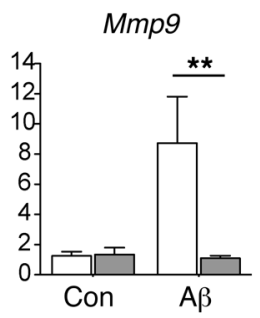

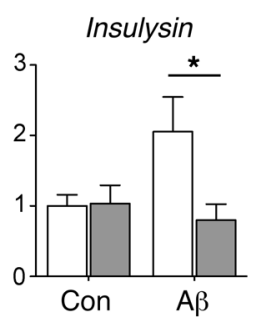

Figure 1. EP2 signaling modulates inflammatory responses to $A \boldsymbol{\beta}_{42}$ oligomers in an age-dependent manner. (A) Peritoneal macrophages of young and aged mice (4 and 21 months, respectively) were stimulated with $A \beta_{42}$ oligomers $(5 \mu \mathrm{M})$. Ep2 mRNA was induced at 4 hours in response to $A \beta_{42}$ oligomers in aged macrophages ( $n=5-6$ per group; effect of $A \beta_{42}$, $P=0.016$, ANOVA). (B) Young and aged peritoneal macrophages were stimulated with $A \beta_{42}$ oligomers $(5 \mu \mathrm{M})$ or vehicle, with or without the EP2 agonist butaprost $(1 \mu \mathrm{M})$, and qPCR was performed at 4 hours ( $n=5-6$ per group). Aged - but not young - macrophages increased expression of inflammatory genes in response to $A \beta_{42}$ oligomers (effect of $A \beta_{42}$ in aged macrophages, Cox2, $P=0.013$; Inos, $P=0.0056$; gp91phox,$P=0.023 ; p 67^{\text {phox }}, P=0.003 ; p 47^{\text {phox }}$, $P=0.013$ ), and these responses were amplified with butaprost costimulation. (C) Butaprost enhanced $A \beta_{42}$ oligomer-mediated IL-1 $\beta$ generation in aged macrophages at 6 hours by ELISA ( $n=3$ per group, effect of $A \beta_{42}, P=0.0006$ ). (D) EP2 agonist suppressed MCP-1 and MIP-1 $\alpha$ generation ( $n=5$ per group; effect of butaprost for MCP-1, $P=0.0062$; effect of $A \beta_{42}$ and butaprost for MIP-1 $\alpha, P<0.0001$ ). (E) Expression of Neprilysin, Mmp9, and Insulysin mRNA was induced in aged macrophages with $A \beta_{42}$ oligomers, but suppressed with butaprost $(n=10-11$ per group; for Neprilysin, effect of $A \beta_{42}, P=0.0005$, effect of butaprost, $P=0.0014$; for $M m p 9$, effect of $A \beta$ and butaprost, both $P<0.05$; for Insulysin, effect of interaction, $P<0.05$ ). (A-E) ${ }^{*} P<0.05$ ${ }^{* *} P<0.01,{ }^{* *} P<0.001$ as indicated, Bonferroni post-hoc. microglial chemokine generation and chemotaxis, clearance of $A \beta$ peptides, resolution of innate inflammatory responses to $A \beta_{42}$, and trophic factor generation and signaling. We further demonstrate that ablation of microglial EP2 signaling prevents cognitive impairment and loss of synaptic proteins in AD model mice.

\section{Results}

EP2 signaling exerts age-associated and opposing effects on proinflammatory and chemokine gene expression in response to $A \beta_{42}$ oligomers. $\mathrm{A} \beta_{42}$ oligomers are early inducers of synaptic and neuronal injury in $\mathrm{AD}$ model mice (27). In addition to their direct disruption of synaptic function, $\mathrm{A} \beta_{42}$ oligomers generate a robust NF- $\mathrm{kB}-$ and IFNregulatory factor 1-dependent (IRF1-dependent) inflammatory response (28) that can secondarily injure synapses and neurons. To determine the function of EP2 signaling in young and aged immune responses to oligomeric $A \beta_{42}$ peptides, we assayed the effects of the selective EP2 agonist butaprost in macrophages stimulated with $\mathrm{A} \beta_{42}$ oligomers; because yields of viable microglia suitable for culture experiments are very low when harvested from adult brain (29), we examined peritoneal macrophages (which share many properties with microglia) harvested from both young (4 months) and aged (21 months) C57B6/J mice. We found that Ep2 mRNA was significantly induced in aged but not young macrophages in response to $\mathrm{A} \beta_{42}$ oligomers $(5 \mu \mathrm{M}$; Figure $1 \mathrm{~A})$. Consistently, $\mathrm{A} \beta_{42}$ oligomers induced a robust inflammatory transcriptional response in aged but not young macrophages that was further increased by costimulation with $1 \mu \mathrm{M}$ butaprost (Figure 1B). A $\beta_{42}$-induced increases in IL-1 $\beta$ generation and secretion were further amplified with butaprost (Figure 1C and Supplemental Figure 1A; supplemental material available online with this article; doi:10.1172/JCI77487DS1), which suggests that myeloid EP2 signaling increases inflammasome generation of IL-1 $\beta$. Conversely, expression of the chemokines MCP-1 and MIP-1 $\alpha$, which are involved in myeloid cell recruitment to sites of injury, was suppressed with butaprost both basally and with $A \beta_{42}$ stimulation (Figure 1D and Supplemental Figure 1, B and C). Finally, expression of $A \beta$ peptide clearance enzymes, notably Neprilysin, Insulysin, and Mmp9, was also suppressed with EP2 activation in $\mathrm{A} \beta_{42}$-stimulated macrophages (Figure 1E). Taken together, these findings demonstrate an age-dependent pattern of gene regulation by EP2 signaling in the context of $\mathrm{A} \beta_{42}$-induced innate immune responses, with induction of proinflammatory factors (including IL-1 $\beta$, COX-2, iNOS, and NADPH oxidase subunits) and suppression of chemokines and proteases important in microglial migration and $\mathrm{A} \beta_{42}$ oligomer clearance.

EP2 ablation increases microglial chemotaxis to nascent amyloid plaques in the APP-PS1 mouse model. Given that EP2 signaling strongly suppressed generation of chemokines in response to oligomeric $A \beta_{42}$, we investigated whether EP2 signaling nega- 
A

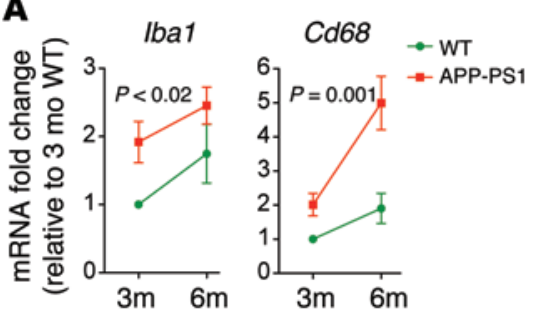

D
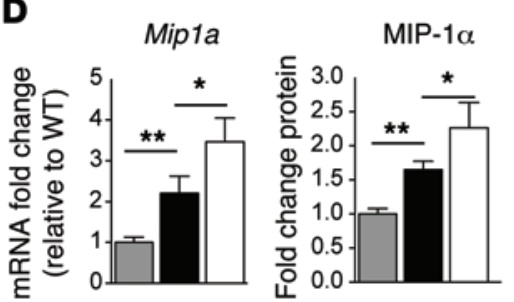

B

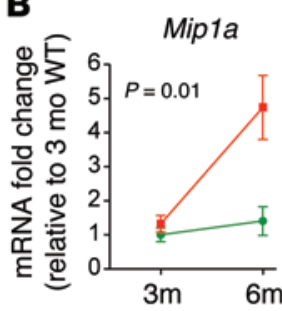

E

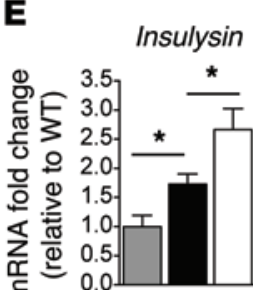

C

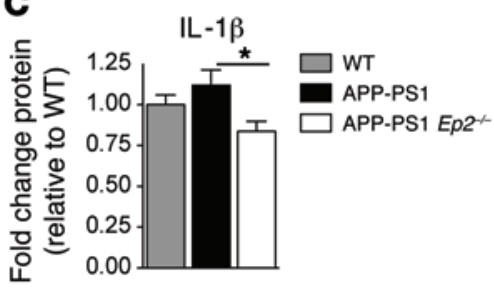

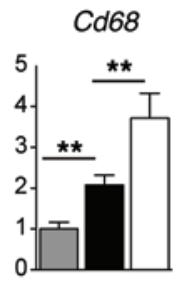

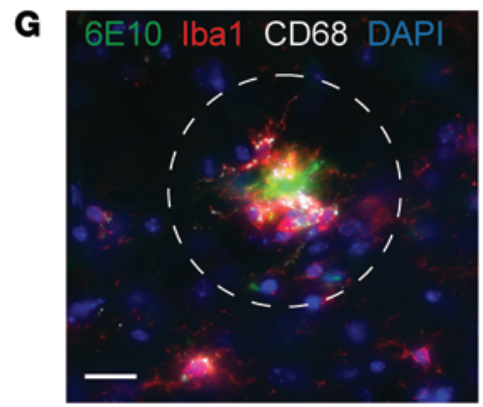

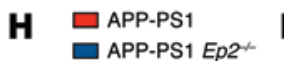 Microglia Microglia
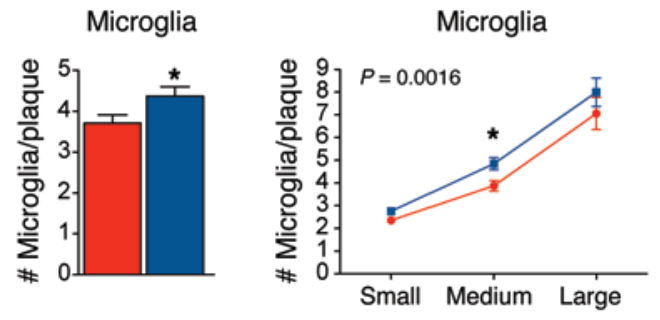

J

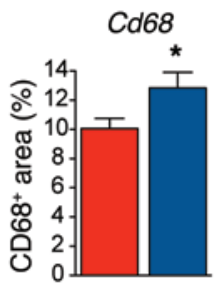

Figure 2. Microglial chemotaxis to early accumulating A $\beta$ peptides is enhanced with Ep2 deletion in 4- to 5-month-old APP-PS1 mice. (A) qPCR demonstrated preplaque increases in hippocampal Iba1 and Cd68 (effect of genotype, Iba1, $P<0.02 ; C d 68, P=0.001 ; n=5-9$ per group). (B) Mip1a was increased early (effect of genotype, $P=0.01 ; n=5-9$ per group). (C) Decreased cerebral cortical IL-1 $\beta$ levels in APP-PS1 Ep2 ${ }^{-1-}$ versus APP-PS1 mice ( $n=14-19$ per group). Increased (D) Mip1a hippocampal mRNA ( $n=8-13$ per group) and MIP-1 $\alpha$ cerebral cortical protein $(n=13-21$ per group) and (E) Insulysin ( $n=8-13$ per group) in APP-PS1 Ep2 ${ }^{-/-}$versus APP-PS1 brains. (F) Increased Iba1 and Cd68 mRNA in preplaque 4-month-old APP-PS1 Ep2 $2^{-/-}$hippocampus ( $n=8-13$ per group). (G) Representative $A \beta$ plaque with surrounding IBA1 ${ }^{+}$and $C D 68^{+}$microglia from 5 -month-old hippocampus. Dashed circle indicates area around plaques used to quantify numbers of microglia and CD68 immunofluorescence. Scale bar: $25 \mu \mathrm{m}$. (H) Microglia around hippocampal plaques in 5-monthold mice; average IBA1+ microglia number increased with Ep2 deletion ( $n=4-7$ mice per group, total 102-122 plaques per group). (I) Ep2 deletion enhanced microglial recruitment to early plaques (effect of genotype, $P=0.0016$; effect of plaque size, $P<0.0001$; post-hoc $P<0.05$ for medium-sized plaque). Small, $<250 \mu \mathrm{m}^{2}$; medium, 250-575 $\mu \mathrm{m}^{2}$; large, $>575 \mu \mathrm{m}^{2}$. (J) Increased CD68 staining in periplaque hippocampal microglia of APP-PS1 Ep2 $2^{-/-}$mice ( $n=5-6$ mice per genotype, total 26-48 plaques per genotype). ${ }^{*} P<0.05,{ }^{* *} P<0.01$, ANOVA with Bonferroni post-hoc (A, B, and I) or Student's $t$ test (C-H and J).

tively affects microglial chemotaxis in vivo, using the APP-PS1 (APPSwe-PS1 $\triangle E$ 9) mouse model of familial AD. This transgenic line coexpresses the human $\mathrm{APP}^{\mathrm{Swe}}$ and $\mathrm{PS}^{1 \mathrm{E} 9}$ mutant transgenes and exhibits $A \beta$ peptide plaque deposition beginning at 5 months, with later onset of synaptic loss and spatial memory deficits after 8-9 months of age (30). We found that before significant $A \beta$ plaque accumulation had begun at 3 months, a robust microglial response was already underway, characterized by increased expression of the cytoskeletal protein Iba1 and the lysosomal glycoprotein $C d 68$ (Figure 2A); the chemokine Mip1a began increasing at 3 months, with a significant rise by 6 months (Figure 2B), presumably in response to accumulating $A \beta_{42}$ peptide oligomers and fibrils.

Global deletion of Ep2 in APP-PS1 mice (referred to herein as APP-PS1 $\left.E p 2^{-/}\right)$reduced cerebral cortical IL-1 $\beta$ protein at 5 months of age and increased hippocampal Mip1a mRNA, cortical MIP-1 $\alpha$ protein, and Insulysin mRNA (Figure 2, C-E), consistent with the in vitro findings shown in Figure 1. Interestingly, APP-PS1 Ep2 $2^{-1-}$ hippocampus exhibited significantly increased levels of $I b a 1$ and
Cd68 compared with control APP-PS1 hippocampus (Figure 2F), suggestive of an altered activation state in microglia lacking Ep2.

We then tested whether EP2-mediated regulation of MIP-1 $\alpha$ was associated with altered chemotaxis of microglia to sites of accumulating $A \beta$ peptides in APP-PS1 hippocampus (Figure $2 \mathrm{G}$ ). At 5 months, a time point at which $A \beta$ peptides begin to accumulate in this model, deletion of Ep2 in the APP-PS1 Ep2 $2^{--}$mice increased microglial recruitment to nascent $A \beta$ plaques, as assayed by quantification of IBA $1^{+}$microglia surrounding newly formed $A \beta$ plaques (Figure $2 \mathrm{H}$ ). Additional quantification of microglia around small, medium, and large $A \beta$ plaques demonstrated a significant effect of genotype and plaque size (Figure 2I). Levels of CD68 were significantly increased in APP-PS1 Ep2-/- mice (Figure 2J). We did not find differences in levels of $A \beta_{42}$ between genotypes at this age, presumably because $A \beta$ peptide accumulation is very low at 5 months, in contrast to later ages of 8-9 months, at which APP-PS1 $E p 2^{-/-}$mice exhibited a reduction in cumulative $A \beta$ peptide load (Supplemental Figure 2). Taken together, these findings suggest 
A
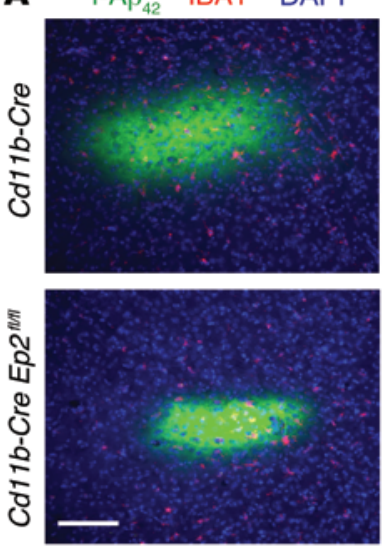

B

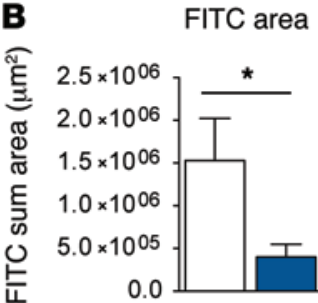

IBA1
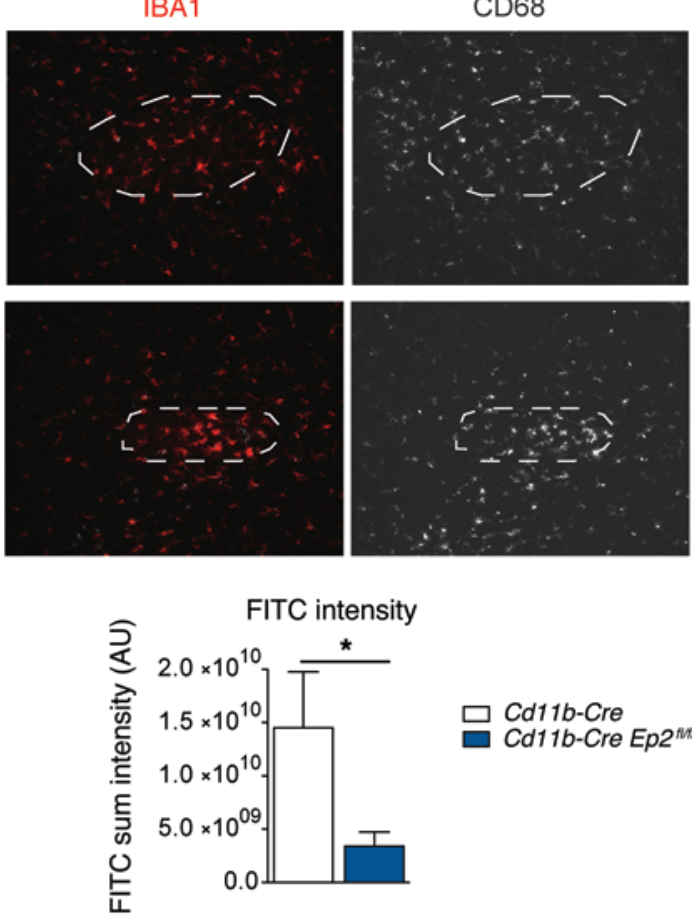

Figure 3. Conditional deletion of microglial Ep2 accelerates clearance of intracortically injected A $\beta$ peptide. $C d 11 b$-Cre Ep $2^{f / f l}$ and $C d 11 b$-Cre mice (17 months old, $n=6-7$ per group) underwent intracortical injection of $185 \mathrm{pmol}$ fibrillar $A \beta_{42}$ or vehicle, and cortical tissue was analyzed 48 hours later. (A) Representative images of sections at the injection site containing $A \beta_{42}$ deposits visualized by FITC fluorescence and immunostained for microglial markers IBA1 and CD68. Scale bar: $100 \mu \mathrm{m}$. (B) Remaining FITClabeled $A \beta$ peptide at 48 hours after injection was significantly reduced in $\mathrm{Cd} 11 \mathrm{~b}-\mathrm{Cre} \mathrm{Ep} 2^{f / f l}$ versus $C d 11 b$-Cre cerebral cortex $\left({ }^{*} P<0.05\right.$, Student's $t$ test). (C) Relationship between intensity of microglial markers IBA1 and CD68 and the remaining $\mathrm{FITC}^{+}$area showed a significant correlation (Cd11b-Cre, IBA1, $r^{2}=0.88$, CD68, $r^{2}=0.93$; Cd11b-Cre Ep2 $2^{f / f 1}$, IBA1, $r^{2}=0.88$, CD68, $r^{2}=0.88$ ) Correlation of microglial IBA1 and CD68 levels with remaining $\mathrm{FITC}^{+}$area showed significant difference between genotypes $(P<0.0001)$, with higher IBA1 and CD68 intensity present in Cd11b$\mathrm{Cre} E p 2^{f / / f l}$ sections per given $\mathrm{FITC}^{+}$area.
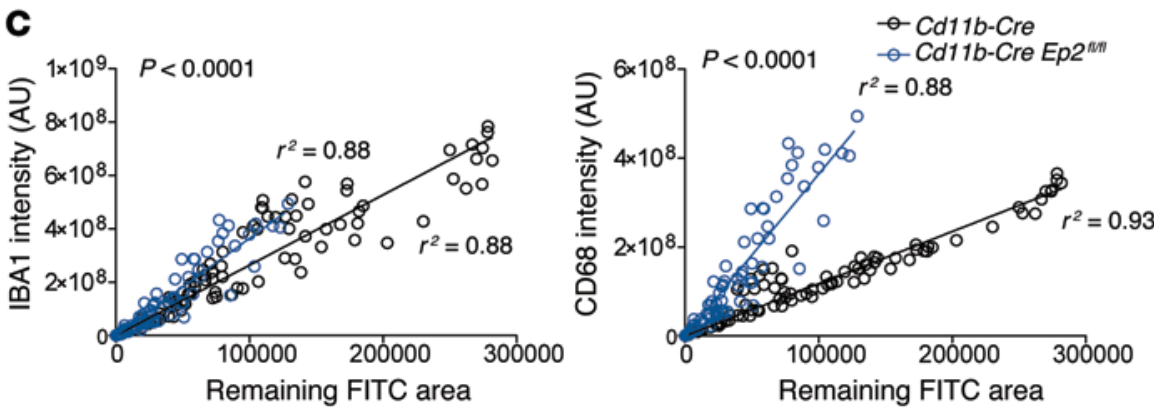

that at the earliest stages of pathology in APP-PS1 mice, inhibition of EP2 signaling resulted in beneficial microglial responses to accumulating $A \beta$ peptides, with suppressed proinflammatory IL-1 $\beta$ generation and increased MIP- $1 \alpha$ expression and microglial chemotaxis to sites of $A \beta$ peptide accumulation.

Conditional deletion of Ep2 in microglia increases microglial activation and clearance of $A \beta$ peptides. We next used a microglial conditional knockout strategy to directly examine microglial EP2 function in chemotaxis and clearance of $\mathrm{A} \beta$ peptide in vivo. The $C d 11 b$-Cre recombinase line leads to excision of floxed sequences in cells of the myeloid lineage, including monocytes, macrophages, and microglia, and has been successfully used to examine neuroinflammatory responses in brain $(26,29,31)$. We injected 17-month-old Cd11b-Cre Ep $2^{f / f l}$ and control littermate Cd11b-Cre mice intracortically with FITC-conjugated fibrillar $A \beta_{42}$ peptides and examined them 48 hours later to quantify microglial activation and remaining fluorescent $A \beta_{42}$ peptide (Figure $3 \mathrm{~A}$ ). The remaining FITC $^{+}$staining area and intensity, quantified in a blinded fashion (see Methods), indicated that clearance of $A \beta_{42}$ peptides was significantly higher in $C d 11 b$-Cre $E p 2^{f / f l}$ mice than in $C d 11 b$ Cre controls (Figure 3B). Although absolute numbers of microglia were not counted, intensities of IBA1 and CD68 were increased in $C d 11 b$-Cre Ep $2^{f / f l}$ cortex for any given level of remaining FITC ${ }^{+}$ $\mathrm{A} \beta_{42}$ area $(P<0.0001$ between slopes; Figure $3 \mathrm{C})$. These findings support a primary function of microglial Ep2 in suppressing recruitment and activation of microglia that clear $A \beta_{42}$ peptides chronically in the APP-PS1 model and acutely after intracortical $\mathrm{A} \beta_{42}$ peptide injection.

Microglial EP2 signaling regulates inflammatory and noninflammatory pathways in vivo in response to $A \beta_{42}$ peptides. Although microglia function in innate immune brain responses, they are also intimately associated with neurons and synapses and perform essential nonimmune functions important to normal neural function. These include maintaining structural plasticity by pruning and elimination of synapses, clearing apoptotic cells, and generating trophic and neurogenic factors (32). Our findings thus far suggested a harmful function of microglial EP2 signaling both in vitro and in vivo in models of $A \beta_{42}$ inflammation, with potentiation of proinflammatory responses, suppression of immune cell trafficking to sites of $A \beta$ peptide accumulation, and suppression of $A \beta$ peptide clearance. To identify additional functions of microglial EP2 signaling, we turned to an unbiased approach and examined microglial-specific gene expression in response to i.c.v. injection of $A \beta_{42}$ peptides. $A \beta_{42}$ peptide injection i.c.v. not only generates 
A Microglial genes

Cd11b-Cre Ep2 $2^{\text {mII }}+\mathrm{A} \beta$ vs. Cd11b-Cre + A $\beta$ (55)

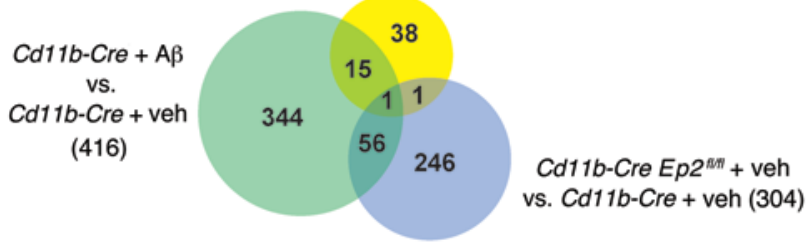

\section{B KEGG pathways} (Cd11b-Cre + A $\beta$ vs. Cd11b-Cre + veh)

mmu04060:Cytokine-cytokine receptor interaction mmu04672:Intestinal immune network for IgA production mmu05330:Allograft rejection

mmu04062:Chemokine signaling pathway

mmu05310:Asthma

mmu05320:Autoimmune thyroid disease

mmu04514:Cell adhesion molecules (CAMs)

mmu05416: Viral myocarditis

mmu04620:Toll-like receptor signaling pathway

mmu05322: Systemic lupus erythematosus

mmu05340:Primary immunodeficiency

mmu04640:Hematopoietic cell lineage

mmu04621:NOD-like receptor signaling pathway

mmu05414:Dilated cardiomyopathy

mmu04010:MAPK signaling pathway

mmu04623:Cytosolic DNA-sensing pathway

mmu05332:Graft-versus-host disease

mmu04670:Leukocyte transendothelial migration

mmu04940:Type I diabetes mellitus

mmu04115:p53 signaling pathway

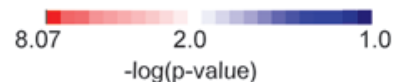

D
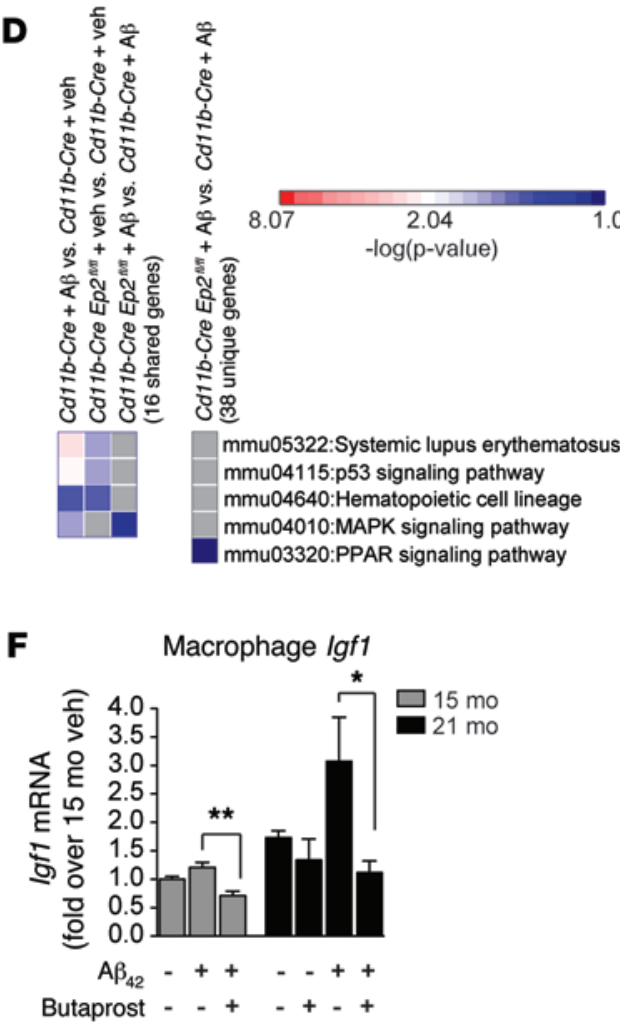

$\mathbf{E}$
C

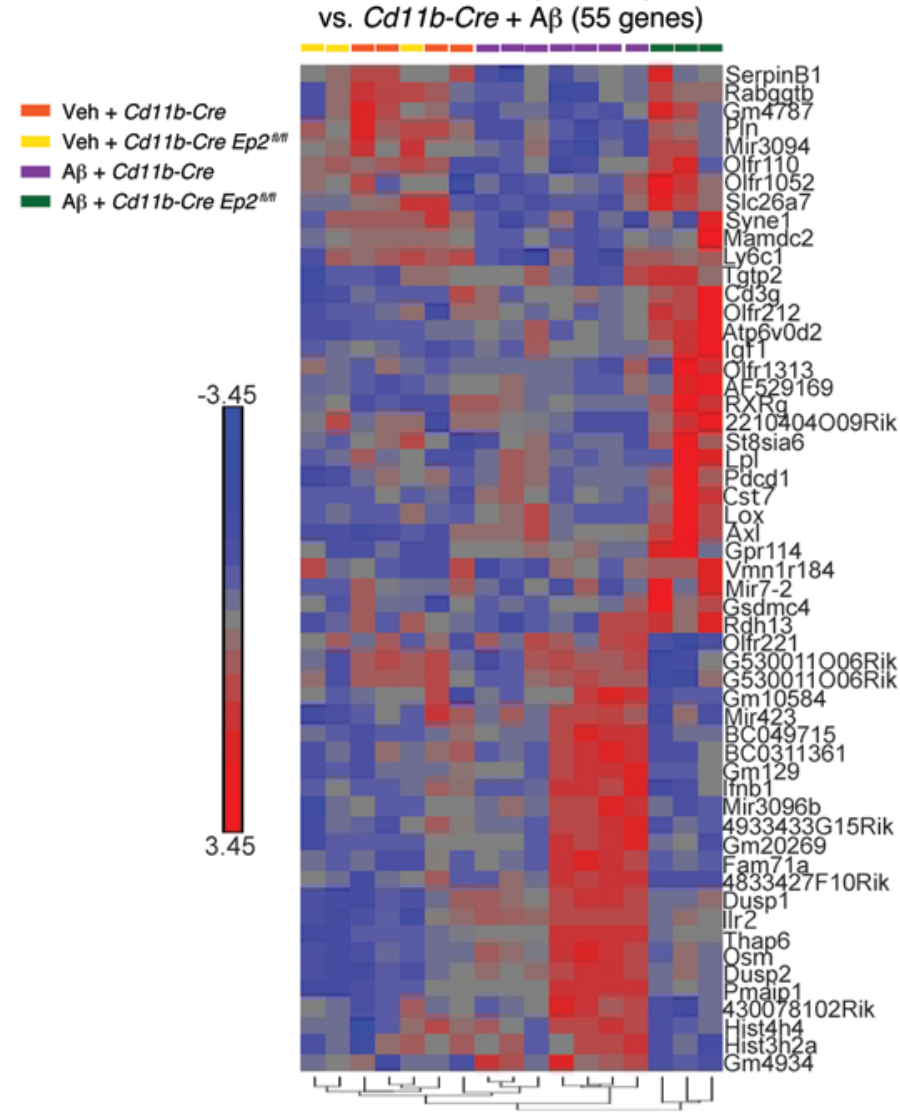

$\square$ Cd11b-Cre $\square$ Cd11b-Cre Ep2 $2^{\text {nn }}$
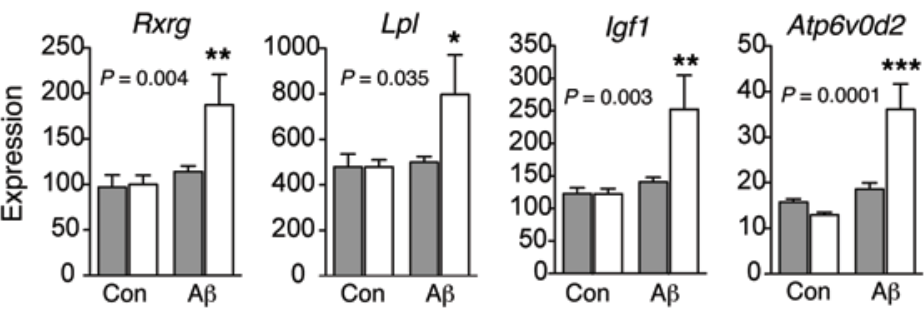

Figure 4. Microglial EP2 signaling regulates distinct immune and non-immune pathways in response to i.c.v. $\mathbf{A} \boldsymbol{\beta}_{42^{2}}$. Adult microglia were harvested for microarray analysis from brains of 8-month-old Cd11b-Cre and Cd11b-Cre Ep $2^{f / f / f}$ mice 48 hours after i.c.v. administration of either vehicle or $A \beta_{42}$ fibrillar peptides. (A) Venn diagram of the 3 comparisons. (B) KEGG pathways significantly enriched in $A \beta_{42}$ - versus vehicle-treated Cd11b-Cre mice. (C) Hierarchical clustering of 55 genes differentially regulated in the i.c.v. $A \beta_{42}$-treated $C d 11 b$-Cre Ep $2^{f / f l}$ group versus the i.c.v. $A \beta-$ treated Cd11b-Cre group. (D) KEGG pathways shared between comparisons, with the PPAR $\gamma$ pathway represented in 38 uniquely regulated genes in the $C d 11 b-C r e ~ E p 2^{f / f l}$ i.c.v. $A \beta_{42}$ versus $C d 11 b-C r e$ i.c.v. $A \beta$ comparison. Pathways that are not shared are shaded gray. (E) Expression levels for regulated genes compared between $C d 11 b$ - $C r e E p 2^{f / f l}$ and $C d 11 b$ - $C r e$ groups treated with i.c.v. $A \beta_{42}$ include genes encoding PPAR $\gamma$ signaling (Rxrg and $L p l$ ), microglial lysosomal (Atp6vOd2), and trophic (Igf1) proteins. $P$ values for main effect of $A \beta_{42}$ treatment are shown in the figure (Bonferroni post-hoc between genotypes, ${ }^{*} P<0.05,{ }^{* *} P<0.01,{ }^{* *} P<0.001 ; n=3-7$ per group). (F) Butaprost suppressed expression of Igf1 in peritoneal macrophages of 15- and 21-month-old mice stimulated with $A \beta_{42}$ oligomers at 4 hours ( ${ }^{*} P<0.05,{ }^{*} P<0.01$, Student's $t$ test; $n=5-6$ per group). 
a robust, long-lasting innate immune response to $A \beta_{42}$ (21), but also disrupts memory consolidation (33), and thus represents a model in which to test effects of microglial EP2 on transcriptional responses and functional outcomes that are altered in response to $\mathrm{A} \beta_{42}$ peptides. We performed microarray analysis on RNA isolated from adult microglia from 8-month-old Cd11b-Cre Ep $2^{f / / f}$ and $C d 11 b$-Cre mice 48 hours after injection of i.c.v. A $\beta_{42}$ peptides.

We examined 3 genetic comparisons (absolute fold change $\geq 1.5, P<0.05$; Figure 4A). Gene Ontology (GO) expression analysis for the comparison between $\mathrm{A} \beta$ - versus vehicle-injected $C d 11 b$ Cre mice showed the Immune System Process as the most highly enriched (enrichment score, 94.42). Expression levels of microglial Ep2 were increased 1.30-fold $(P=0.013)$ at 48 hours after i.c.v. $\mathrm{A} \beta$ in the $C d 11 b$-Cre control genotype. Unsupervised hierarchical clustering of differentially expressed genes revealed a striking distinction between the i.c.v. $A \beta$ and i.c.v. vehicle treatment groups (Supplemental Figure 3A). Ingenuity Pathway Analysis (IPA) of upstream regulatory transcription factors demonstrated 2 major nodes of inflammatory gene regulation, $N f k b$ and Irf7 (Supplemental Figure 3B). In this comparison, Cox2, which is upstream of EP2, was highly induced in vivo in microglia from i.c.v. $A \beta_{42}$-treated mice (Supplemental Figure 3C). Application of IPA revealed that many $\mathrm{A} \beta$-regulated genes were also regulated by $\mathrm{COX}-2$ and $\mathrm{PGE}_{2}$ (Supplemental Figure 3B).

Functional annotation of the 416 transcripts differentially expressed in $\mathrm{A} \beta$ - versus vehicle-treated $C d 11 b$-Cre mice was carried out using the Database for Annotation, Visualization and Integrated Discovery (DAVID; see Methods). This analysis revealed 20 Kyoto Encyclopedia of Genes and Genomes (KEGG) pathways that were significantly enriched, almost all of which corresponded to inflammatory signaling networks (Figure 4B). Comparison of A $\beta$-treated $C d 11 b$-Cre Ep2 $2^{f / f l}$ versus $C d 11 b$-Cre mice revealed 55 regulated genes (Figure $4 \mathrm{~A}$ ), and hierarchical clustering of these genes across conditions demonstrated a clear segregation of $\mathrm{A} \beta$-regulated genes in Cd11b-Cre Ep $2^{f / f}$ mice (Figure 4C). Comparison of KEGG pathways revealed shared pathways between the $\mathrm{A} \beta$-treated $C d 11 b$-Cre and vehicle-treated $C d 11 b$-Cre groups and between the vehicle-treated $C d 11 b$-Cre $E p 2^{f / f l}$ and vehicle-treated Cd11b-Cre groups (Figure 4D). The complete set of enriched KEGG pathways in the Cd11b-Cre Ep $2^{f / f l}$ versus $C d 11 b$-Cre comparison included cell cycle, proteolysis, and immune pathways (Supplemental Figure 3D).

Interestingly, the majority of differentially regulated genes in the $\mathrm{A} \beta$-treated $C d 11 b$-Cre $E p 2^{A / f / A}$ versus $\mathrm{A} \beta$-treated $C d 11 b$-Cre comparison were not regulated by $A \beta$, but were specifically changed with microglial Ep2 deletion (38 genes; Figure 4A). This suggested that rather than simply reversing $A \beta_{42}$-induced inflammatory changes, $\mathrm{Cd11b}$-Cre Ep $2^{A / / A}$ microglia engaged alternative response pathways. Functional annotation of these 38 genes using DAVID revealed an enrichment of PPAR signaling pathway genes, including retinol dehydrogenase-13 (Rdh13), retinoid $\mathrm{X}$ receptor $\gamma(R x r g)$, and lipoprotein lipase ( $L p l$ ) (Figure 4, D and E). Rdh13 (1.78-fold increase) participates in the endogenous synthesis of retinoic acid (RA) that binds and activates RXR subunits. PPAR $\gamma /$ RXR heterodimers inhibit proinflammatory gene expression (reviewed in ref. 34) and increase phagocytosis of A $\beta$ peptides (35). $R x r g$, along with RXR and LXR heterodimers, increases expression of the cholesterol transporter ABCA1 (36) and apolipoprotein E (ApoE) (37), proteins that enhance proteolytic degradation of soluble $A \beta$ peptides $(38,39)$; recent studies indicate that administration of the FDA-approved RXR agonist bexarotene (Targretin) reduces interstitial levels of soluble $A \beta$ peptides and rescues behavioral deficits in AD model mice $(40,41)$. $L p l$, which functions in lipoprotein remodeling and cholesterol transport, and whose expression is driven by RA and RXR/LXR transcriptional activity, was increased 1.52-fold with deletion of microglial Ep2. The upregulation of these genes is suggestive of induction of antiinflammatory and $A \beta$-clearing nuclear hormone receptor signaling genes in the response of EP2-deficient microglia to $\mathrm{A} \beta_{42}$ peptides in vivo. Added to this was the induction of $\mathrm{H}^{+}$transporting ATPase (Atp6vOd2; 1.92-fold induction; Figure 4E), a proton pump expressed in lysosomes of myeloid cells. Atp6rOd2 participates in degradation of proteins targeted to the lysosome (42), suggestive of a potential role in $\mathrm{A} \beta_{42}$ degradation.

Insulin-like growth factor 1 (IGF1) is upregulated in vivo in microglia derived from Cd11b-Cre Ep $2^{f / f}$ brains. In addition, we found an unexpected increase in Igf1 mRNA levels in microglia derived from i.c.v. $\mathrm{A} \beta$-treated $\mathrm{Cd} 11 \mathrm{~b}$-Cre $E \mathrm{E} 2^{f / f t}$ mice (Figure $4 \mathrm{E}$ ). Whereas at the organismal level, reduced IGF1 signaling increases longevity (43), at the cellular level, IGF1 promotes cell survival through the PI3K/AKT pathway and RasGTPase/RAF-1/MEK pathways, and in brain, IGF1 signaling promotes synaptogenesis, neurogenesis, angiogenesis, and neuroprotection (44). Although IGF1 receptors are expressed on all cell types in the CNS, in general, IGF1 is synthesized in the liver and is transported to the brain bound to IGF1 binding proteins. Exceptions include postnatal brain development, where microglia transiently express IGF1 that supports developing layer V neurons (45), and following brain injury, where microglia express IGF1 and astrocytes and neurons increase IGF receptor expression (44). Validation of the EP2-dependent regulation of IGF1 was carried out in aged primary macrophages, where Igf1 mRNA expression was found to be suppressed by the EP2 agonist butaprost (Figure 4F). Taken together, our unbiased analyses indicated the activation of multiple beneficial pathways in Ep2-deficient microglia in vivo, including antiinflammatory nuclear hormone, $\mathrm{A} \beta$ clearing, and trophic pathways. Moreover, these pathways were activated in parallel with suppression of the proinflammatory response (see below).

Cd11b-Cre Ep $2^{A / f}$ mice stimulated i.c.v. with $A \beta_{42}$ show increased IGF1 receptor signaling and reduced inflammation. We next tested whether microglial Ep2 deletion increased IGF1 signaling following stimulation with i.c.v. $A \beta_{42}$. Binding of IGF1 to its tyrosine kinase receptor (IGF1R) leads to phosphorylation of IGF1R and recruitment of multiple scaffold proteins, including insulin receptor substrates 1-4 (IRS-1-IRS-4) and Src homology 2 domain-containing transforming protein 1 (44), which bind with different time courses to phosphorylated IGF1R to transduce IGF1 signaling. Here, $C d 11 b$-Cre Ep $2^{f / / t}$ and $C d 11 b$-Cre mice were treated i.c.v. with vehicle or $\mathrm{A} \beta_{42}$ as above, and hippocampi were analyzed 48 hours later for phosphorylation of IGF1R (Figure 5, A and B, and Supplemental Figure 4, A and B). Quantification of immunoprecipitated total and phosphorylated IGF1R (p-IGF1R) demonstrated a significant increase in the $\mathrm{p}-\mathrm{IGF1R} /$ total IGF1R ratio in Cd11b-Cre $E p 2^{A / f l}$ versus $C d 11 b$-Cre mice treated with i.c.v. $A \beta_{42}$ (Figure $5 \mathrm{~B}$ ). 

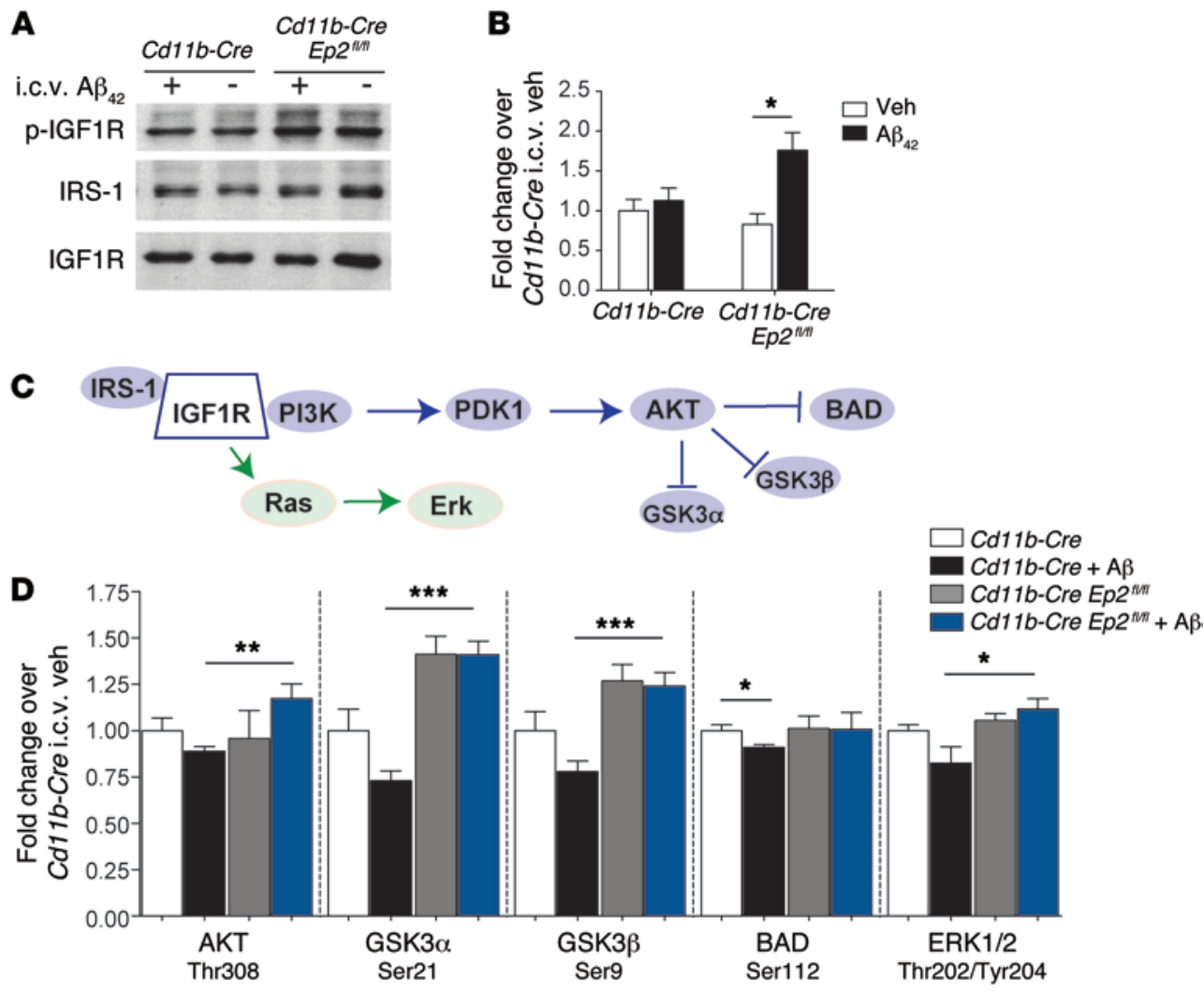

Figure 5. Inhibition of microglial EP2 signaling increases brain IGF1/PI3K signaling after i.c.v. $\mathbf{A}_{42}$ administration. (A) $C d 11 b-C r e$ and $C d 11 b-C r e ~ E p 2^{f / f f l}$ mice were administered vehicle or $A \beta_{42}$ peptides i.c.V., and hippocampi were examined for IGF1R activation at 48 hours. Hippocampal lysates were immunoprecipitated with anti-IGF1R antibody and blotted against p-Tyr to detect p-IGF1R, IRS-1, and total IGF1R. A representative blot is shown. (B) Increased p-IGF1R/ total IGF1R ratio in Cd11b-Cre Ep2 ${ }^{f / f / f}$ hippocampus after i.c.v. $A \beta_{42}$ administration ( $n=6-9$ per group; ${ }^{*} P<0.05$, Student's $t$ test). (C) Diagram of the IGF1R/ AKT pathway. (D) Quantification of phosphoproteins downstream of PI3K/AKT in cerebral cortex showed increased AKT and ERK1/2 signaling in i.c.v. A $\beta$ treated Cd11b-Cre Ep2 $2^{f / f l}$ mice ( $n=6-9$ per group; ${ }^{*} P<0.05,{ }^{* *} P<0.01,{ }^{* * *} P<0.001$, Student's $t$ test).

We also measured levels of IRS-1, one of several scaffolding proteins that bind to phosphorylated IGF1R, and found a trend toward increased binding in the Cd11b-Cre Ep $2^{f / f}$ genotype (Supplemental Figure 4C). No changes in levels of total IGF1R were noted between genotypes (Supplemental Figure 4D).

To examine the effect of increased IGF receptor signaling in $C d 11 b$-Cre Ep2 $2^{f / f l}$ mice, we quantified phosphorylation levels of candidate proteins known to be phosphorylated downstream of IGF1R/PI3K in cerebral cortex (Figure 5, C and D, and Supplemental Figure 4, E and F). Levels of AKT phosphorylated by PDK1 at Thr308 and levels of p-ERK1/2 were significantly increased in i.c.v. A $\beta$-stimulated $C d 11 b$-Cre Ep $2^{f / f}$ mice compared with $C d 11 b$ Cre controls. Similarly, phosphorylation of proteins directly targeted by AKT, including GSK $3 \alpha$ and GSK3 $\beta$, also increased in i.c.v. A $\beta$-treated Cd11b-Cre Ep $2^{A / A}$ mice. Phosphorylation of BAD at Ser112 and ERK1/2 at Thr202/Tyr204 was suppressed in i.c.v. A $\beta-$ treated $C d 11 b$-Cre mice, but preserved in i.c.v. $\mathrm{A} \beta$-treated $C d 11 b$ Cre $E p 2^{f / f}$ mice. Taken together, these data indicate that increased IGF1R signaling in $C d 11 b$-Cre Ep $2^{f / f l}$ mice resulted in enhanced $\mathrm{AKT} / \mathrm{ERK}$ signaling in the setting of $\mathrm{A} \beta$ stimulation in vivo.

As AKT signaling promotes beneficial antiinflammatory effects, we examined the effect of microglial Ep2 deletion on the neuroinflammatory response to i.c.v. A $\beta$. Levels of cytokines and chemokines were measured using Luminex multiplex assay in hippocampi isolated from $C d 11 b$-Cre Ep $2^{f / f l}$ and $C d 11 b$-Cre mice treated or not with i.c.v. $A \beta_{42}$ at 48 hours. In $C d 11 b$-Cre controls, i.c.v. $A \beta_{42}$ broadly significantly upregulated 22 factors, compared with 7 factors differentially regulated in Cd11b-Cre Ep $2^{f / f l}$ mice (Figure 6A and Supplemental Figure 5); these included the proinflammatory cytokines IL-1 $\alpha$, IL-1 $\beta$, IL-17A, and IL-6. The chemokines MIP-1 $\alpha$, MIP-1 $\beta$, and RANTES were also highly regulated by EP2 in this model, but in the opposite manner to that found in the APP-PS1 model, which suggests that regulation of chemokines by EP2 in vivo is context specific and may differ between chronic and acute $A \beta_{42}$ stimulation. In the i.c.v. $A \beta_{42}$ model, $A \beta_{42}$ is administered acutely and initiates a robust inflammatory response, in contrast to transgenic expression in the APP-PS1 model, which leads to chronic, low-level production of $A \beta$ peptides. Alternatively, lower chemokine generation in the i.c.v. $A \beta_{42}$ model may reflect a more benign inflammatory milieu of $C d 11 b$-Cre Ep $2^{f / f / f}$ brain at the 48-hour time point. Taken together, the findings of increased brain IGF1 signaling in combination with reduced production of inflammatory ILs suggest a beneficial effect of microglial Ep2 deletion in the context of $\mathrm{A} \beta_{42}$-generated immune responses.

Conditional deletion of microglial Ep2 prevents a functional deficit in novel object recognition (NOR). Neuroinflammatory responses can significantly impair cognitive function via effects of cytokines, proteases, and oxidative stress on synapses and neurons. We test- 
A

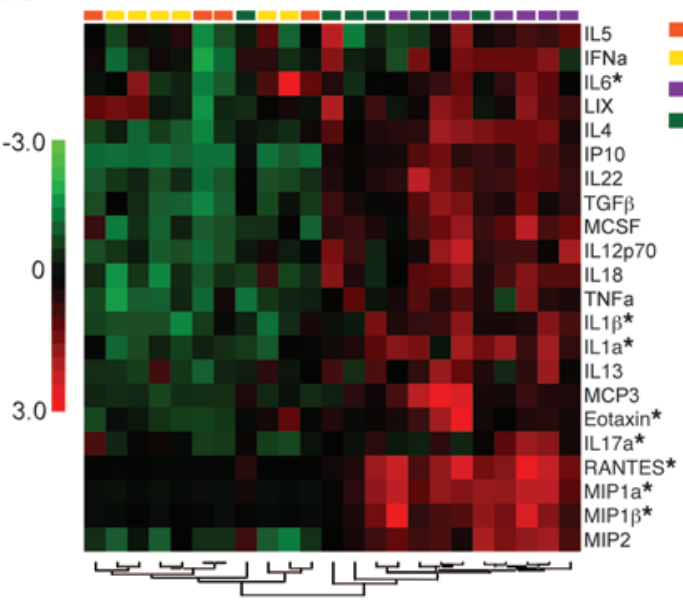
Cd11b-Cre Ep2 $2^{m n}+$ veh

- Cd11b-Cre + A

- Cd11b-Cre Ep2 $2^{n \pi}+\mathrm{A} \beta$
Cd11b-Cre + veh

Figure 6. Inhibition of microglial EP2 signaling decreases inflammation and rescues memory following i.c.v. $A \beta_{42}$ administration. (A) Cluster analysis producing separation for genotype and treatment is shown for 22 immune factors in cerebral cortex that were significantly upregulated in hippocampus 48 hours after i.c.v. $A \beta_{42}$. Asterisks denote factors that were differentially regulated by microglial EP2 (see Supplemental Figure 5). (B) Overview of the NOR memory test, with the novel object shaded green. (C) DI (comparing 0-hour training session with 24-hour recognition session) demonstrated normal memory consolidation in control i.c.v. vehicle-treated $C d 11 b$-Cre and Cd11b-Cre Ep $2^{f / / f l}$ mice, and absence of memory consolidation in the i.c.v. $A \beta_{42}$-treated Cd11b-Cre cohort, which was significantly rescued by microglial Ep2 deletion in the i.c.v. $A \beta_{42}$-treated Cd11b-Cre Ep2 $2^{f / / f l}$ cohort ( $n=8-15$ mice per group; ${ }^{* *} P<0.01,{ }^{*} P<0.05$, paired 1-tailed Student's $t$ test).

test), in contrast to i.c.v. $A \beta_{42}$-injected Cd11b-Cre mice, which were not able to recognize the old from the new object at 24 hours. Importantly, this $A \beta_{42}$-induced NOR deficit was prevented in Cd11b-Cre Ep $2^{A / f /}$ mice $(P<0.01$, paired $t$ test). Taken together, our findings support a highly beneficial effect of microglial Ep2 ablation, resulting in suppression of proinflammatory responses, increased signaling through the IGF1R pathway, and prevention of NOR memory deficits.

Conditional deletion of microglial Ep2 reduces spatial memory deficits in APP-PS1 mice. We then tested the effect of deletion of microglial Ep2 in the APP-PS1 model, in which progressive amyloid accumulation and inflammation lead to synaptic loss and hippocampal-dependent memory deficits. Male APP-PS1 Cd11b-Cre Ep $2^{f / / t}$ mice and APP-PS1 Cd11b-Cre controls were aged to 9 months, the time point at which spatial memory deficits begin in this line. Hippocampal-dependent spatial memory per-

ed whether microglial Ep2 negatively affects memory function in the setting of $\mathrm{A}_{42}$-mediated inflammation. Control experiments examining locomotor, anxiety, and Y-maze performance did not show differences between $C d 11 b$-Cre and Cd11b-Cre Ep $2^{f / f l}$ mice (Supplemental Figure 6). Next, we used NOR, a memory task that relies on the innate preference of mice to spend more time with a novel rather than a familiar object, which is significantly disrupted in the i.c.v. $\mathrm{A} \beta_{42}$ model (33). NOR requires the normal function of the perirhinal and entorhinal cerebral cortex and the hippocampus. As illustrated in Figure 6B, on day 1 after i.c.v. injection of either vehicle or $A \beta_{42}$, mice were habituated to an empty arena and later allowed to briefly explore 2 identical objects (training session, 0 hours). After 24 hours, mice were again put in the arena; however, one of the objects used during training was replaced by a novel object. Recognition memory (recognition session, 24 hours) of the old versus new object was assessed as the discrimination index (DI), the ratio of time spent exploring the old object to time spent exploring both objects. A DI of $\sim 50 \%$ is characteristic of the training session, where there is no preference for either of the 2 objects; with normal memory consolidation, decreased DI during the recognition session reflects less time spent with the old object and more time exploring the new object, as was shown for the i.c.v. vehicle-injected Cd11b-Cre and Cd11b-Cre Ep $2^{f / f l}$ groups (Figure 6C). These control mice performed normally $(P<0.05$, paired $t$ formance in the radial arm maze (RAM) was tested (Figure 7A). Behavior in the RAM was quantified over the last 3 days of a 6-day period in which mice were evaluated for their ability to locate a new rewarded choice arm after visiting a previously rewarded sample arm. For the first 3 days of testing, no significant differences were observed for any genotype. However, the second 3 days of testing showed a significant difference between APP-PS1 $C d 11 b$-Cre and APP-PS1 Cd11b-Cre Ep2 $2^{R / A}$ mice for mean number of errors per trial $(P<0.05$, APP-PS1 Cd11b-Cre versus APP-PS1 Cd11b-Cre Ep2 $2^{f / f} ; P=0.089$, APP-PS1 Cd11b-Cre versus Cd11bCre; Figure 7A). APP-PS1 Cd11b-Cre mice also showed increased latency to make a correct choice compared with Cd11b-Cre mice $(P<0.05)$, and this was partially improved with deletion of microglial Ep2 ( $P=0.075$, APP-PS1 Cd11b-Cre versus APP-PS1 Cd11b-Cre $E 2^{A / f / f} ;$ Figure 7A).

We then assessed effects of microglial Ep2 deletion on synaptic integrity by quantifying levels of candidate synaptic proteins (Figure 7, B and C). The loss of synaptophysin correlates with progression of cognitive decline in AD development (46); moreover, studies in transgenic mouse AD models have demonstrated that presynaptic proteins are disrupted early during amyloid accumulation $(21,47)$, with loss of postsynaptic markers occurring at more advanced stages of pathology (48). At 8-9 months, we found a decrease in levels of the presynaptic proteins synaptophysin and 
A

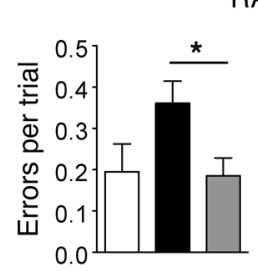

RAM

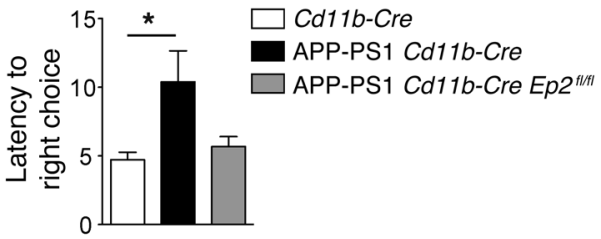

B

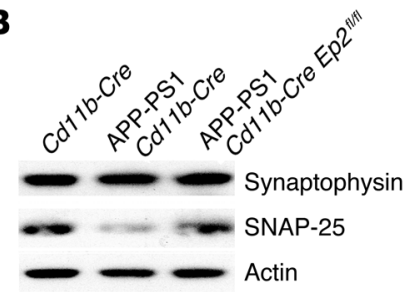

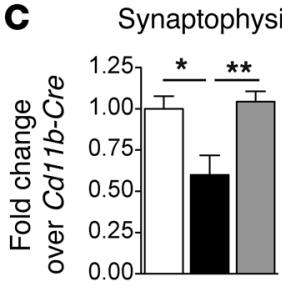

D

Hippocampal lgf1

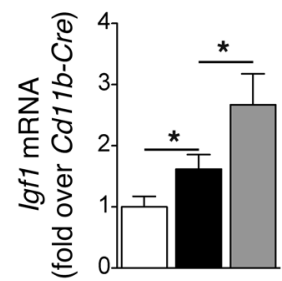

SNAP-25

PSD-95

GLUA1
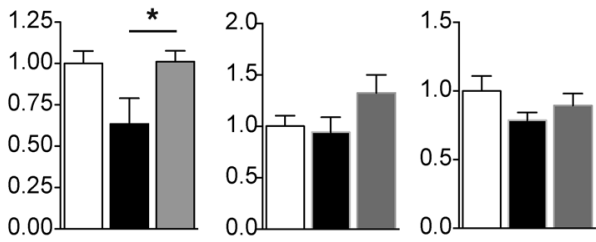
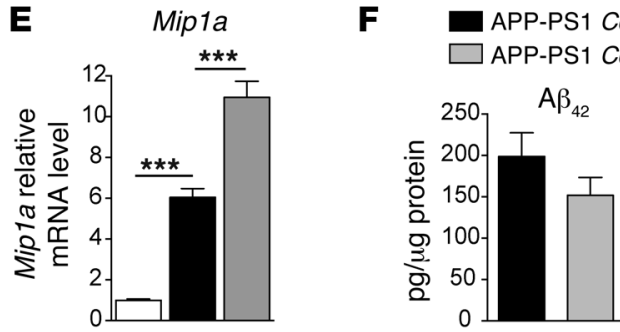

G

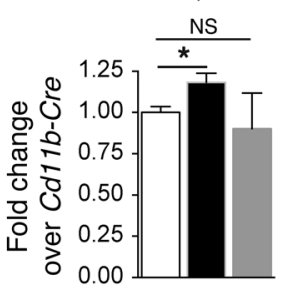

PDK1 (Ser241)

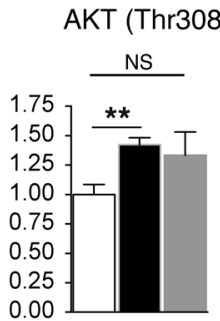

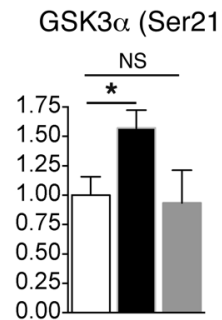

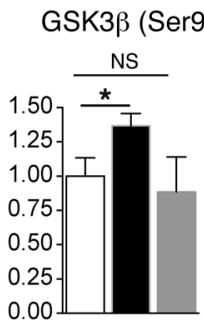

BAD (Ser112)

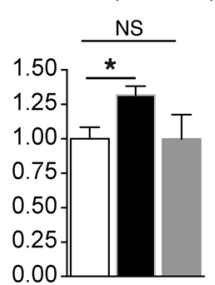

Figure 7. Effects of microglial Ep2 deletion in 9-month-old male APP-PS1 mice on spatial memory performance, presynaptic protein levels, and PI3K/AKT signaling. (A) Cd11b-Cre, APP-PS1 Cd11b-Cre, and APP-PS1 Cd11b-Cre Ep2 ${ }^{f / / 1}$ cohorts were assessed for spatial memory performance in the RAM, using mean errors per trial and latency to make a correct choice as outcome measures ( $n=7-11$ per group). Whereas APP-PS1 Cd11b-Cre Ep2f/ffl and Cd11b-Cre animals made similar numbers of errors per trial during the course of testing, APP-PS1 Cd11b-Cre mice made significantly more ( ${ }^{*} P<0.05$, Mann-Whitney U test). Cd11b-Cre and APP-PS1 Cd11b-Cre Ep2 ${ }^{f / f l}$ mice also made the correct choice more quickly than did APP-PS1 Cd11b-Cre mice $(P<0.05)$. (B and C) The loss of synaptophysin and SNAP-25 observed in APP-PS1 Cd11b-Cre mice was reversed in APP-PS1 Cd11b-Cre Ep2 $2^{f / f l}$ mice ${ }^{*} P<0.05$, ${ }^{* *} P<0.01$, Student's $t$ test; $n=5-6$ per group). The postsynaptic proteins PSD-95 and GLUA1 were not changed. (D) Increased Igf1 mRNA in APP-PS1 Cd11b-Cre Ep2f//f versus APP-PS1 Cd11b-Cre mice and in APP-PS1 Cd11b-Cre versus Cd11b-Cre mice ( $n=6-10$ per group; ${ }^{*} P \leq 0.05$, Student's $t$ test). (E) Increased Mip1a mRNA expression in APP-PS1 Cd11b-Cre Ep2 $2^{f / f l}$ versus APP-PS1 Cd11b-Cre hippocampus ( $n=8-13$ per group; ${ }^{* *} P<0.0001$; Student's $t$ test). (F) Cerebral cortex from APP-PS1 Cd11b-Cre and APP-PS1 Cd11bCre Ep2 $2^{f / f}$ mice was assayed for $A \beta_{42}$ levels by ELISA ( $P=0.17$, Mann-Whitney 2-tailed $t$ test; $n=5-6$ per group). (G) Quantification of PI3K/AKT phosphoproteins in cerebral cortex of $C d 11 b-C r e$, APP-PS1 Cd11b-Cre, and APP-PS1 Cd11b-Cre Ep2 $2^{f / f l}$ mice showed significant induction of the AKT signaling pathway in APP-PS1 Cd11b-Cre mice that was absent in APP-PS1 Cd11b-Cre Ep $2^{f / f / l}$ mice $\left({ }^{*} P<0.05,{ }^{* *} P<0.01\right.$, Student's $t$ test; $n=4-5$ per group).

SNAP-25 in APP-PS1 Cd11b-Cre mice compared with Cd11b-Cre controls that was rescued by deletion of microglial Ep2; no changes were demonstrated in the postsynaptic proteins PSD-95 and GLUA1 in either group. Thus, the loss of presynaptic markers was prevented with deletion of microglial Ep2 in the APP-PS1 Cd11bCre Ep $2^{A / f l}$ cerebral cortex.

Consistent with the i.c.v. $A \beta_{42}$ model, Igfl mRNA levels were

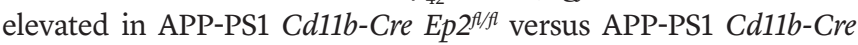
hippocampus (Figure 7D). We also assessed levels of chemokine expression and total $\mathrm{A} \beta_{42}$ in APP-PS1 Cd11b-Cre and APP-PS1 Cd11bCre $E 2^{2 / / f l}$ mice. For chemokine expression, we found significantly

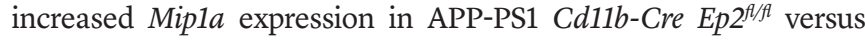
APP-PS1 Cd11b-Cre 9-month-old male mice (Figure 7E); this was consistent with the increased Mip1a expression observed in APPPS1 Ep2 ${ }^{--}$mice and in vitro data in aged macrophages (Figures 1 and 2). In APP-PS1 Cd11b-Cre Ep $2^{A / f l}$ cerebral cortex, we found a $23.7 \%$ decrease in mean total $\mathrm{A} \beta_{42}$ levels $(P=0.17$; Figure $7 \mathrm{~F})$, which was not as marked as the effect in APP-PS1 $E p^{2--}$ mice, in which mean cortical levels of $A \beta_{42}$ were reduced by $36.5 \%$ at 8-9 months $(P<0.01$; Supplemental Figure 2). The lack of significant decline in $\mathrm{A} \beta_{42}$ levels with microglial $E p 2$ deletion may be due to incomplete excision of floxed sequences, and we have previously demonstrated 


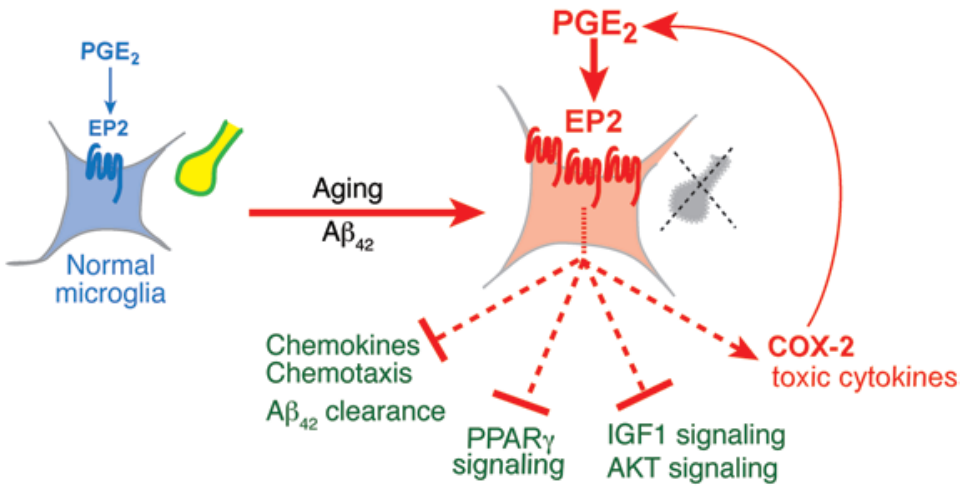

Figure 8. Functions of $\mathrm{PCE}_{2}$ EP2 signaling in healthy and aged/ $\mathbf{A} \boldsymbol{\beta}_{42}$-stimulated microglia. Basal EP2 signaling in healthy microglia regulates normal homeostatic effects on cell cycle, cytoskeletal, and immune functions (Supplemental Figure 3D) and maintenance of healthy synapses (yellow). With aging and/or $A \beta_{42}$ accumulation, microglial EP2 signaling is upregulated, increasing proinflammatory gene expression and suppressing beneficial chemokine production and chemotaxis, A $\beta$ clearance, nuclear hormone PPAR $\gamma$ signaling, and IGF1/AKT signaling. A major target upregulated by EP2 is COX-2 (Supplemental Figure 3B), which drives additional $\mathrm{PCE}_{2}$ production, perpetuating toxic EP2 inflammatory signaling. In combination, these EP2 regulated effects contribute to synaptic degeneration (gray) and functional memory impairment. that the $C d 11 b$-Cre recombinase line is approximately $50 \%$ efficient in excising floxed Ep2 sequences (26). Incomplete excision of floxed sequences is common in many Cre-mediated systems, in which recombinase activity frequently results in cell-specific knockdown of gene expression. Alternatively, it is possible that chronic accumulation of $A \beta$ peptide by 9 months of age in this transgenic model may overwhelm the ability to clear $A \beta$, in spite of the beneficial effects of microglial Ep2 deletion.

To examine the effect of chronic suppression of microglial EP2 signaling in the APP-PS1 model, we again examined phosphorylation of candidate proteins in the IGF1R/PI3K pathway in cerebral cortex of 9-month-old APP-PS1 Cd11b-Cre Ep2 $2^{f / f}$, APP-PS1 Cd11bCre, and Cd11b-Cre mice (Figure $7 \mathrm{G}$ and Supplemental Figure 7). In this chronic model of $A \beta$ stimulation, there was a significant induction of AKT signaling, with increased p-PDK1 (Ser241), p-AKT (Thr308), p-GSK2 $\alpha$ (Ser21), p-GSK3 $\beta$ (Ser9), and p-BAD (Ser112) in APP-PS1 Cd11b-Cre versus Cd11b-Cre mice. Interestingly, APP-PS1 Cd11b-Cre Ep $2^{f / / A}$ mice exhibited abolished AKT signaling induction, which suggests that in the context of microglial Ep2 deletion, IGF1R/PI3K signaling was more similar to $C d 11 b$-Cre levels. Moreover, as many pathways feed into the AKT pathway, the normalization of AKT signaling to Cd11b-Cre levels in APP-PS1 Cd11b-Cre $E p 2^{f / f l}$ brain may also reflect chronic effects of multiple beneficial microglial functions activated as a result of microglial Ep2 deletion.

\section{Discussion}

Microglial activities represent critical lines of defense against the development of neurodegenerative disease; microglia clear misfolded proteins, elaborate trophic and regenerative factors, and regulate and terminate toxic inflammation. Recent studies point to a steady decline of these normal microglial functions in aging and in $\mathrm{AD}$. In $\mathrm{AD}$, microglia not only lose their capacity to clear $\mathrm{A} \beta$ peptides but also develop a persistent proinflammatory phenotype that does not resolve, accelerating neuronal and synaptic injury $(49,50)$. In this study, using in vitro and in vivo genetic strategies, we found that microglial EP2 activity negatively regulates multiple and distinct beneficial functions that are critical in opposing the harmful effects of accumulating $A \beta_{42}$. Together, our findings in distinct mouse models of $A \beta$ inflammation demonstrated that deletion of microglial Ep2 restores chemotaxis, $A \beta$ clearance, regulation of inflammatory responses, and trophic factor generation and signaling (Figure 8), with behavioral correlates of preventing cognitive deficits and loss of synaptic proteins.
In vitro, aged but not young macrophages increased EP2 expression in response to $\mathrm{A} \beta_{42}$ oligomers. This interesting agedependent regulation was associated with a robust immune response to $A \beta_{42}$ that was significantly enhanced with coadministration of EP2 agonist. The observed difference in inflammatory responses to $A \beta_{42}$ between young and aged macrophages is consistent with observations demonstrating that innate immune responses increase with age, in part through increased activation of NF- $\mathrm{KB}$-driven inflammatory pathways (51). Moreover, the age dependence of EP2 upregulation and EP2-driven inflammatory gene expression is highly relevant to the pathogenesis of $\mathrm{AD}$, for which the primary risk factor is age. We also noted a converse suppressive effect of EP2 signaling on expression of chemokines and $\mathrm{A} \beta$-degrading enzymes, proteins that are beneficial in controlling extracellular levels of $A \beta$ peptides in vivo. Thus, in response to $A \beta_{42}$ oligomers, EP2 signaling upregulated expression of deleterious oxidative and proinflammatory factors and reduced expression of beneficial chemotactic and proteolytic genes. This dual regulatory function suggests that microglial EP2 signaling may be playing a harmful role in vivo in response to early $A \beta_{42}$ generation and accumulation. This was confirmed in young 5-month-old APPPS1 Ep2 $2^{-/-}$mice, which exhibited not only increased chemotaxis of microglia to sites of nascent amyloid plaques, but increased levels of $\mathrm{A} \beta$ proteases and CD68. Moreover, in Cd11b-Cre Ep $2^{f / f l}$ mice, microglial deletion of Ep2 significantly accelerated clearance of intracortically injected $A \beta$ peptides.

The opposing regulation of MIP- $1 \alpha$ by A $\beta$ peptides and by EP2 signaling is particularly interesting. Inflammatory chemokines such as MIP- $1 \alpha$, MIP- $1 \beta$, and MCP- 1 are $\beta$ chemokines that are important in recruitment of monocytic cells, including macrophages and microglia. Upon binding to their G proteincoupled receptors (CCR1 and 5 for MIP-1 $\alpha$ ), these chemokines initiate cytoskeletal reorganization and cellular migration. Of all chemokines, MIP- $1 \alpha$ in particular was highly induced by 6 months in the APP-PS1 model, suggestive of a specific role for microglial MIP- $1 \alpha$ in the inflammatory response to $A \beta$ peptides. Moreover, in addition to its well-established function of inducing chemotaxis of immune cells, MIP- $1 \alpha$ can also activate immune cells to produce inflammatory cytokines. Thus, the suppression of MIP-1 $\alpha$ expression coupled to the induction of proinflammatory responses in the context of A $\beta$ stimulation and EP2 signaling is unexpected, but likely suggests a complex transcriptional regulation of MIP- $1 \alpha$ and cytokine release. Nonetheless, 
the functional outcome of EP2 signaling in the context of A $\beta$ stimulation - worsening of the inflammatory response through increased proinflammatory gene expression, as well as suppression of MIP- $1 \alpha$ and microglial chemotaxis to sites of $A \beta$ peptide accumulation - is detrimental.

To explore the broader regulatory role of microglial EP2 signaling in response to $A \beta_{42}$, we used an unbiased approach and identified a class of genes upregulated independently of $A \beta_{42}$ in $C d 11 b$-Cre Ep $2^{f / f l}$ mice. Among these genes were the nuclear hormone transcriptional pathway components $R x r g, R d h 13$, and $L p l$, components of PPAR and RA pathways that promote antiinflammatory, trophic, and phagocytic microglial activities and that are highly beneficial in AD models (35). RXR $\gamma$ binds the clinical compound bexarotene and increases levels of ApoE 4 and ABCA1 lipidator important for $\mathrm{A} \beta$ peptide clearance by ApoE; activation of RXR/LXRs by bexarotene may lower interstitial soluble $A \beta$ peptide levels and improve cognitive performance $(40,52)$. The regulation of RXR $\gamma$ in microglia is also interesting in the context of the circadian clock, which is modulated by retinoid receptors via regulation of BMAL1 transcription (53). Thus, it is conceivable that EP2 regulation of $\mathrm{RXR} \gamma$ could modulate the forward arm of the clock. Recent studies have linked deficient BMAL:CLOCK/NPAS2 activity to disrupted energy homeostasis, aging, and neurodegeneration (54-56), which suggests that the prominent increase in EP2 signaling in aging myeloid cells shown in Figure 1 may negatively affect myeloid cellular clock function.

Intriguingly, Ep2-deficient microglia responded to $A \beta_{42}$ peptides by upregulating IGF1; in brain, this growth factor is not only potently neuroprotective, neurogenic, and antiinflammatory, but also enhances synaptic and neuronal plasticity and improves cognitive function (44). Although IGF1 can be produced in most tissues, most of it is generated by the liver and released into the circulation. Circulating IGF1 decreases with age and in AD; moreover, $\mathrm{AD}$ is characterized by insulin resistance and decreased IGF1R signaling (57). In mouse models of $A D$, circulating serum IGF1 can accelerate clearance of $A \beta$ peptides (58). In our studies of mice acutely administered i.c.v. $A \beta_{42}$, deletion of microglial Ep2 significantly increased microglial IGF1R signaling in hippocampus. Examination of protein phosphorylation downstream of IGF1R and PI3K after $A \beta$ administration revealed marked induction in PI3K/AKT signaling in Cd11b-Cre Ep $2^{f / f l}$ mice, which suggests that deletion of Ep2 in microglia preserves and enhances the AKT signaling response to a noxious stimulus, in this case $A \beta$ peptides. AKT signaling is beneficial and antiinflammatory; consequently, Cd11b-Cre Ep $2^{f / f l}$ mice exhibited a reduced inflammatory response and were also able to function normally in the NOR memory task. Taken together, these findings argue for a beneficial protective effect of microglial Ep2 deletion in the context of an acute $A \beta$ stimulus.

Ablation of microglial Ep2 signaling similarly elicited a beneficial effect in the context of chronic $A \beta$ stimulation, notably in aging 9-month-old APP-PS1 Cd11b-Cre Ep $2^{f / A l}$ mice. Spatial memory testing was improved in APP-PS1 Cd11b-Cre Ep $2^{f / f t}$ mice, as were levels of presynaptic proteins. APP-PS1 Cd11b-Cre Ep $2^{f / f l}$ animals also demonstrated increased levels of IGF1 and MIP- $1 \alpha$ expression. Interestingly, in this chronic model, AKT signaling was broadly induced in APP-PS1 Cd11b-Cre mice compared with
Cd11b-Cre controls; however, deletion of microglial Ep2 normalized the APP-PS1 Cd11b-Cre Ep $2^{f / f l}$ phosphoprotein levels toward $C d 11 b$-Cre levels. This suggests that chronic microglial Ep2 deletion led to a more benign inflammatory and oxidative environment; this, in addition to the trophic and antiinflammatory effects of chronic IGF1R/PI3K signaling, might be expected to blunt a reactive increase in AKT signaling.

Taken together, our findings suggest a broad effect of conditional deletion of Ep2 in microglia in mouse models of AD. This conclusion was based on the selectivity of the $C d 11 b$ promoter-driven expression of Cre recombinase in brain to microglia, as demonstrated by Boillee et al.: crosses of Cd11b-Cre mice to Rosa26-lacZ mice showed Cre recombination in the CNS that was restricted to microglia (31). In a previous study using this Cre recombinase line, we observed approximately $50 \%$ reduction of floxed Ep2 sequences in microglia and in peritoneal macrophages (26). Peripherally, $\mathrm{CD} 11 \mathrm{~b}$ protein is normally expressed in monocytes and tissuespecific macrophages, and to a lesser extent in subpopulations of granulocytes, mature B lymphocytes, and $\mathrm{CD}^{+} \mathrm{T}$ lymphocytes $(59,60)$. Because Cd11b-Cre recombinase will also reduce levels of peripheral myeloid EP2, we cannot exclude a potential contribution of peripheral myeloid cell Ep2 deletion to our present findings. There is increasing evidence of communication between the periphery and the CNS, particularly in experimental models of aging, where circulating factors can alter hippocampal neurogenesis, synaptic plasticity, and cognitive function $(61,62)$. Validation with cell-specific Cre recombinase lines that are selectively expressed only in brain microglia or peripherally in macrophages/ monocytes will be very helpful in parsing out the relative contributions of these cell types to models of $\mathrm{AD}$ and aging.

In conclusion, our data demonstrate that microglial EP2 suppresses multiple beneficial functions that are essential to combat the toxic effects of $A \beta_{42}$ peptides on synapses and memory function. These findings suggest that microglial EP2 activity hastens pathological progression to AD. By virtue of its broad regulatory effect on beneficial microglial functions, inhibition of inflammatory EP2 signaling may be a promising strategy to restore healthy microglial function, arrest the progression of $A \beta_{42}$-driven pathology, and prevent development of AD.

\section{Methods}

Animals. All strains were in the C57BL/6 background. APP-PS1 mice

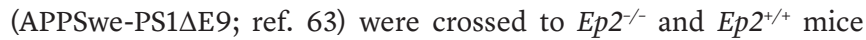
(originally from R. Breyer, Vanderbilt University, Nashville, Tennessee, USA; refs. 64, 65). Cd11b-Cre mice (31), provided by G. Kollias (Alexander Fleming Biomedical Sciences Research Center, Vari, Greece) and D. Cleveland (UCSD, La Jolla, California, USA), efficiently excise floxed sequences in microglia and macrophages (26, 29, 31). C57BL/6 Ep2 $2^{f /+}$ mice have been previously described (26). All mice were housed in an environment controlled for lighting (12-hour light/12-hour dark cycle), temperature, and humidity, with food and water available ad libitum.

Macrophage cell cultures. Peritoneal macrophages were harvested from young (3-5 months) and aged (19-21 months) female mice (except for MIP-1 $\alpha$ ELISA, for which males were used). Viable and healthy macrophages can be obtained from both young and aged mice and survive well in culture; this is not the case for adult- 
derived microglia, which may not survive consistently in culture and yield highly variable data. In addition, macrophage yields are significantly higher per mouse than are microglia yields, thus minimizing the number of mice required for the study. Mice were injected with $1.5 \mathrm{ml} 3 \%(\mathrm{w} / \mathrm{v}$ ) thioglycollate medium (BD Diagnostic Systems) into the peritoneal cavity, and primary macrophages were isolated 3-4 days later by flushing with ice-cold HBSS (HyClone). Cells were seeded at a density of $2 \times 10^{6}$ cells/well onto 12 -well plates for RNA and $8 \times 10^{4}$ cells/well in 96-well plates for ELISA measurements in DMEM supplemented with $10 \%$ heat-inactivated FBS (HyClone), $100 \mathrm{U} / \mathrm{ml}$ penicillin and streptomycin, and $1 \mathrm{mM}$ sodium pyruvate and maintained at $5 \% \mathrm{CO}_{2}$ at $37^{\circ} \mathrm{C}$. After overnight culture, cells were washed twice with medium to remove nonadherent cells before treatment.

Primary microglia culture. Primary microglia were isolated from the brains of postnatal day 7 C57BL/6J mouse pups obtained from Charles River Laboratories. Primary microglia were isolated using the Neural Tissue Dissociation Kit (P), MACS Separation Columns (LS), and magnetic CD11b Microbeads from Miltenyi Biotec. Microglia were grown in culture for 3-5 days before being treated in each experiment as previously described (26).

Preparation of oligomeric and fibrillar $A \beta_{42}$. Oligomeric and fibril$\operatorname{lar} \mathrm{A} \beta$ peptide species were generated as previously described $(21,66)$.

Quantitative real-time PCR ( $q P C R)$. RNA isolation, cDNA production, and SYBR-Green-based qPCR (QuantiTect SYBR Green Kit; Qiagen) were performed as previously described (29) using the standard curve method and normalizing to $18 S$ and Gapdh. Primers were designed by PrimerQuest (Integrated DNA Technologies) and synthesized by Integrated DNA Technologies. Primer sequences are listed in Supplemental Table 1.

Intracortical and i.c.v. injection of $A \beta_{42}$ peptides. FITC-conjugated fibrillar $\mathrm{A} \beta_{42}$ peptides $(185 \mu \mathrm{M}$; rPeptide) or vehicle were administered intracortically to 17 -month-old female and male Cd11b-Cre and Cd11bCre $E p 2^{f / f / l}$ littermates $(67,68)$. Mice were under isoflurane anesthesia during surgery. $1 \mu \mathrm{l}$ fibrillar $\mathrm{A} \beta_{42}$ (185 pmol) or vehicle was delivered to opposite hemispheres of the cerebral cortex over 10 minutes using a 32-gauge Hamilton syringe; after injection, the needle was left in place for 5 minutes and then withdrawn over 4 minutes to prevent backflow. The stereotaxic injections were placed at the following coordinates from the bregma: mediolateral, $1.2 \mathrm{~mm}$; anteroposterior, $1.2 \mathrm{~mm}$; dorsoventral, $1.5 \mathrm{~mm}$. After injection, each mouse recovered spontaneously on a heated pad. At 48 hours after injection, mice were euthanized, and brains were rapidly harvested. Intracortical $A \beta_{42}$-injected brains were sectioned coronally on a freezing microtome at $40-\mu \mathrm{m}$ intervals. From each mouse, 30 sections were chosen for immunostaining with IBA1 and CD68 antibodies, with sections covering the span of the needle injection track and at least 5 sections anterior and posterior to the injection track. FITC $^{+}$sections (average, 15 per mouse) were analyzed in a blinded manner by FITC intensity or area covered above a threshold level, and mean IBA1 and CD68 intensities were determined within the $\mathrm{FITC}^{+}$area. For i.c.v. A $\beta$ peptide injections, fibrillar $\mathrm{A} \beta$ peptide species ( $40 \mathrm{pmol}$ ) or vehicle (PBS) were administered to $C d 11 b$-Cre and $C d 11 b$-Cre $E p 2^{f / f / A}$ mice as previously described (69). Stereotaxic injections were placed at the following coordinates from the bregma: mediolateral, $-1.0 \mathrm{~mm}$; anteroposterior, $-0.3 \mathrm{~mm}$; dorsoventral, $-2.5 \mathrm{~mm}$. After injection, each mouse recovered spontaneously on a heated pad.
Microglia quantification. Images were acquired in the hippocampal area by a blinded experimenter at $\times 400$ magnification of all identifiable hippocampal plaques with surrounding microglia, and with $5 z$ planes spaced $5 \mu \mathrm{m}$ apart covering the plaque and microglia. A circle centered on the plaque with diameter of $75 \mu \mathrm{m}$ was stamped, and the cropped image was analyzed. IBA $1^{+}$microglia were manually counted going through the $z$ planes and included if they were touching the plaque with either the cell body or processes. Plaques were grouped into small $\left(<250 \mu \mathrm{m}^{2}\right)$, medium $\left(250-575 \mu \mathrm{m}^{2}\right)$ and large $\left(>575 \mu \mathrm{m}^{2}\right)$ sizes. Percent CD68 coverage area above threshold level was determined in the cropped image for the extended focus, including all $z$ planes. 5 sections (average, 10.6 \pm 1.1 plaques) were analyzed per mouse.

Immunostaining. Immunostaining of paraformaldehyde-fixed mouse sections was carried out as previously described (21). Antibodies used include anti-rat CD68 (1:1,000 dilution; AbD Serotec), antirabbit IBA1 (1:2,000 dilution; Wako), and anti-mouse 6E10 for human A $\beta$ (1:2,000 dilution; Covance). Secondary antibodies and detection reagents included donkey FITC-conjugated anti-mouse, Cy3-conjugated donkey anti-rabbit, and DyLight 649-conjugated anti-rat antibody (Jackson ImmunoResearch Laboratories). Biotinylated secondary antibodies (Vector Labs) were used at a dilution of 1:250. Rabbit and mouse sera (Jackson ImmunoLabs) were used as negative controls in place of primary antibodies on adjacent sections.

Image acquisition for immunostaining. Imaging of immunostained sections was done using a Nikon Eclipse E600 microscope (Nikon Instruments) and a Hamamatsu Orca-ER digital camera (Hamamatsu Photonics). Images were analyzed using the measurements module of Volocity 4.3.2 image analysis software (Improvision).

Western analysis and ELISA and multi-antibody array measurement of phosphoproteins. For quantification of phosphorylated IGF1R, hippocampi were homogenized in $10 \mathrm{mM}$ Tris $\mathrm{HCl}, 150 \mathrm{mM} \mathrm{NaCl}, 1 \mathrm{mM}$ EDTA, 1 mM EGTA, 1\% Triton X-100, 1\% Nonidet P-40, 1 mM sodium orthovanadate, and protease inhibitors. After centrifugation to remove insoluble material, supernatants were incubated overnight with bead-conjugated antibody against IGF1R (Santa Cruz), and immunocomplexes were collected with centrifugation; beads were washed in homogenization buffer before separation by SDS-PAGE. Blots were incubated with primary antibodies against phosphotyrosine (4G10, Platinum, Millipore) to detect p-IGF1R; membranes were stripped and reprobed with anti-IGF-I Receptor $\beta$ (Cell Signalling). Levels of MIP- $1 \alpha$ and MCP-1 (R\&D Systems) and IL-1 (BD Biosciences) were measured by ELISA. For quantification of phosphoproteins, $36 \mu \mathrm{g}$ cortical lysates were applied in $60 \mu \mathrm{l}$ to the PathScan AKT Signaling Antibody Array kit (Chemiluminescent Readout, Cell Signaling Technology); the assay was carried out according to the manufacturer's instructions. This assay simultaneously detects 16 phosphoproteins, many belonging to the AKT signaling cascade. Capture antibodies specific for the phosphorylated proteins were spotted on nitrocellulose-coated glass slides, and lysates were incubated overnight at $4^{\circ} \mathrm{C}$. After washing, arrays were incubated with a biotinylated antibody, followed by chemiluminescent film detection. Spot intensities were quantified using ImageJ.

$A \beta$ peptide quantification. $5 \mathrm{M}$ guanidine-extracted $\mathrm{A} \beta_{42}$ peptides were precipitated with ethanol to remove the guanidine, and $A \beta_{42}$ peptides were measured by ELISA as previously described $(22,70)$.

Isolation of primary microglia from adult mouse brain. For isolation of microglia, 8- to 9-month-old Cd11b-Cre and Cd11b-Cre Ep $2^{f / f l}$ mice were administered i.c.v. injection of $A \beta_{42}$ fibrils or saline. At 48 hours 
after surgery, mice were sacrificed by transcardiac perfusion with cold heparinized $0.9 \% \mathrm{NaCl}$. Brains were then removed from the mice and pooled, 2 brains of the same genotype per sample, to ensure adequate cell and RNA yield. The brains were then enzymatically dissociated and isolated using magnetic CD11b microbeads (Miltenyi Biotec) according to the manufacturer's protocol.

RNA isolation and microarray. RNA purification from primary

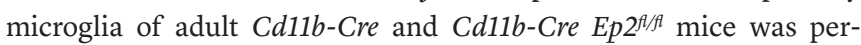
formed using TRIzoL (Life Technologies) followed by the RNeasy Mini Kit (Qiagen). RNA quality was assessed using a BioAnalyzer (Agilent) and determined to be sufficient for microarray analysis (RNA Integrity Number $>7.0$ for all samples). cDNA synthesis, labeling, hybridization, and scanning were performed by the Stanford Protein and Nucleic Acid (PAN) Facility using GeneChip Mouse Gene 2.0 ST arrays (Affymetrix). Microarray data were statistically analyzed using Partek software (Partek) to identify differentially expressed genes between groups by ANOVA using an unadjusted $P$ value of $<0.05$. Genes that had a fold change of $>1.5$ between genotypes were used for unsupervised hierarchical clustering analysis. Database for Annotation, Visualization and Integrated Discovery (DAVID) functional annotation software (version 6.7; NIAID, NIH) was used to identify KEGG molecular pathways significantly overrepresented among lists of differentially expressed genes. Data were deposited in GEO (accession no. GSE57181).

NOR. The NOR task, based on the ability of mice to show preference for novel versus familiar objects when allowed to explore freely (71), is disrupted after i.c.v. administration of $A \beta$ peptides and fibrils (33). NOR was performed during the light cycle. Mice were individually habituated to an open arena $\left(50 \mathrm{~cm} \times 50 \mathrm{~cm}\right.$, dim light, $\left.24^{\circ} \mathrm{C}\right)$ on day 1 . During the subsequent training session, 2 identical objects were placed into the arena, and exploratory behavior was monitored for 5 minutes. On day 2, mice were placed back into the same arena, in which one of the objects used during training was replaced by a novel object of similar dimensions but a different shape/color, and exploratory behavior was monitored for 5 minutes. Digital video tracking (using an infrared camera and vplsi Viewpoint software) of body movements and head position was used to quantify locomotor and exploratory activities around the objects $(2-\mathrm{cm}$ zone around the objects). Exploration behavior was assessed by calculating DI, the ratio of time spent exploring the old object to time spent exploring both objects (expressed as a percentage). A DI of $\sim 50 \%$ is associated with correct training and no object preference; a significant decrease in DI is characteristic of recognition of the novel object. To evaluate memory, comparisons were made for each group between the recognition (24 hours) and training (0 hours) sessions. Paired $t$ tests were performed between time points. Behavioral testing was performed by experimenters blinded to genotype.

$R A M$. The RAM is a spatial memory test that assesses working and reference memory over several consecutive days; the protocol was adopted from that of Clelland et al. (72). Previous experiments demonstrated that no deficits exist in nonmnemonic behaviors (visual and sensory-motor abilities) in APP-PS1 mice (30). Mice were food restricted for 1 week prior to testing and maintained at $85 \%-90 \%$ body weight for the duration of testing, and water was provided ad libitum. All testing occurred at the beginning of the dark phase of the diurnal light/dark cycle or in dim light during the end of the light cycle. The testing apparatus consisted of a wooden 8-arm RAM with Plexi- glas walls, elevated $50 \mathrm{~cm}$ from the floor and surrounded by visual cues. 3 days before testing, mice were habituated to the maze. Day 1 of the habituation phase consisted of a 10-minute group exposure (by cage) to the RAM, in which all arms were unblocked but no food was provided. The second day, mice were individually exposed twice to the maze for 5 minutes, with each arm baited with food rewards. This was repeated on the third day, but with only 3 doors open. Following habituation, mice were tested for their ability to separate sample (familiar) from choice (new) arms in the RAM. Cage order was randomized throughout testing. Mice received 2 trials per day (consisting of 1 sample phase and 1 choice phase) for 6 consecutive days. During the sample phase, all arms except the start arm and the sample arm were blocked off. The mouse was permitted to visit the sample arm and retrieve the food reward. Mice were retrieved from the maze after either (a) spending 10 seconds in the sample arm after retrieving the pellet or (b) exiting the sample arm. During the choice phase, arms in the start and sample (unrewarded) locations and an additional correct (rewarded) location were open. Mice that entered the correct arm were considered to have made correct choices. Mice that made incorrect choices (i.e., entered the sample arm or reentered the start arm) were allowed to self-correct, enter the correct arm, and retrieve the pellet before being removed from the maze. Sample and correct arms were randomized for each combination, such that sample arm was either to the left or right of the start arm, and for each combination, the start arm was located in 1 of 2 locations perpendicular to either the correct or the sample arm.

Statistics. Data are expressed as mean \pm SEM. Statistical comparisons were made with GraphPad Prism software using Student's $t$ test (2-tailed unless otherwise indicated; for 2 groups meeting normal distribution criteria by Shapiro-Wilk normality test), Mann-Whitney U test (for 2 groups not meeting normal distribution criteria), or 2-way ANOVA with Bonferroni multiple-comparison tests (for groups across 2 variables, with multiple comparisons between groups). Linear regression was used to analyze microglial markers in the FITC-A $\beta$ intracortical experiment. The linear model was chosen for goodnessof-fit of the dataset $\left(r^{2}=0.88\right.$ and 0.93). Slopes were compared using $\mathrm{F}$ test. For Luminex multiplex analysis, fluorescence intensities that reached statistical significance with i.c.v. $\mathrm{A} \beta_{42}$ were transformed to relative concentrations (median $z$ score). Cluster analysis (Gene Cluster 3.0 and Java TreeView 1.0.13) produced a separation of samples according to treatment and genotype. For NOR analyses, paired $t$ test was used to compare performance between the 0- and 24-hour time points. Data were subjected to Grubbs' test to identify the presence or absence of outlier data points for exclusion from analysis. For all tests, a $P$ value of 0.05 or less was considered significant.

Study approval. This study was conducted in accordance with NIH guidelines, and protocols were approved by the IACUC at Stanford University.

\section{Acknowledgments}

This work was supported by NIH grant RO1AG030209 (to K.I. Andreasson), NIH grant R21AG033914 (to K.I. Andreasson), Alzheimer's Association (to K.I. Andreasson), Swedish Research Council (to J.U. Johansson), National Science Foundation (to N.S. Woodling), and NRSA grant F31AG039195 (to N.S. Woodling). The authors thank Damien Colas, Bayara Chuluun, Grace Hagiwara, and Craig Heller for assistance in behavioral experiments; 
Frank Longo, Theo Palmer, and Tony Wyss-Coray for use of equipment; and Suraj Pradhan and the Stanford Human Immune Monitoring Center for Luminex measurements.

Address correspondence to: Katrin I. Andreasson, Stanford University School of Medicine, 1201 Welch Road, MSLS P210, Stanford, California 94305, USA. Phone: 650.498.5855; E-mail: kandreas@ stanford.edu.
Jenny U. Johansson's present address is: SRI International, Menlo Park, California, USA.

Nathaniel S. Woodling's present address is: Institute of Healthy Ageing, University College London, London, United Kingdom.

Holden D. Brown's present address is: Brains On-Line LLC, South San Francisco, California, USA.
1. Hebert LE, Scherr PA, Bienias JL, Bennett DA, Evans DA. Alzheimer disease in the US population: prevalence estimates using the 2000 census. Arch Neurol. 2003;60(8):1119-1122.

2. Bateman RJ, et al. Clinical and biomarker changes in dominantly inherited Alzheimer's disease. N Engl J Med. 2012;367(9):795-804.

3. Jack CR, et al. Tracking pathophysiological processes in Alzheimer's disease: an updated hypothetical model of dynamic biomarkers. Lancet Neurol. 2013;12(2):207-216.

4. Sperling RA, et al. Toward defining the preclinical stages of Alzheimer's disease: recommendations from the National Institute on Aging-Alzheimer's Association workgroups on diagnostic guidelines for Alzheimer's disease. Alzheimers Dement. 2011;7(3):280-292.

5. Naj AC, et al. Common variants at MS4A4/ MS4A6E, CD2AP, CD33 and EPHA1 are associated with late-onset Alzheimer's disease. Nat Genet. 2011;43(5):436-441.

6. Bertram L, et al. Genome-wide association analysis reveals putative Alzheimer's disease susceptibility loci in addition to APOE. Am J Hum Genet. 2008;83(5):623-632.

7. Hollingworth $\mathrm{P}$, et al. Common variants at ABCA7, MS4A6A/MS4A4E, EPHA1, CD33 and $\mathrm{CD} 2 \mathrm{AP}$ are associated with Alzheimer's disease. Nat Genet. 2011;43(5):429-435.

8. Guerreiro R, et al. TREM2 variants in Alzheimer's disease. N Engl J Med. 2013;368(2):117-127.

9. Jonsson T, et al. Variant of TREM2 associated with the risk of Alzheimer's disease. N Engl J Med. 2013;368(2):107-116.

10. Lambert JC, et al. Meta-analysis of 74,046 individuals identifies 11 new susceptibility loci for Alzheimer's disease. Nat Genet. 2013;45(12):1452-1458.

11. Lambert JC, et al. Genome-wide association study identifies variants at CLU and CR1 associated with Alzheimer's disease. Nat Genet. 2009;41(10):1094-1099.

12. Raj T, et al. Polarization of the effects of autoimmune and neurodegenerative risk alleles in leukocytes. Science. 2014;344(6183):519-523.

13. Hickman SE, Allison EK, El Khoury J. Microglial dysfunction and defective beta-amyloid clearance pathways in aging Alzheimer's disease mice. J Neurosci. 2008;28(33):8354-8360.

14. Krabbe G, et al. Functional impairment of microglia coincides with Beta-amyloid deposition in mice with Alzheimer-like pathology. PLoS One. 2013;8(4):e60921.

15. in t' Veld BA, et al. Nonsteroidal antiinflammatory drugs and the risk of Alzheimer's disease. NEngl JMed. 2001;345(21):1515-1521.
16. Stewart WF, Kawas C, Corrada M, Metter EJ. Risk of Alzheimer's disease and duration of NSAID use. Neurology. 1997;48(3):626-632.

17. Vlad SC, Miller DR, Kowall NW, Felson DT. Protective effects of NSAIDs on the development of Alzheimer disease. Neurology. 2008;70(19):1672-1677.

18. McGeer PL, Schulzer M, McGeer EG. Arthritis and anti-inflammatory agents as possible protective factors for Alzheimer's disease: a review of 17 epidemiologic studies. Neurology. 1996;47(2):425-432.

19. Montine TJ, et al. Elevated cerebrospinal fluid prostaglandin E2 levels in patients with probable Alzheimer's disease. Neurology. 1998;53(7):1495-1498.

20. Combrinck M, et al. Levels of CSF prostaglandin E2, cognitive decline, and survival in Alzheimer's disease. J Neurol Neurosurg Psychiatry. 2006;77(1):85-88.

21. Shi J, et al. Inflammatory prostaglandin E(2) signaling in a mouse model of Alzheimer disease. Ann Neurol. 2012;72(5):788-798.

22. Liang X, et al. Deletion of the prostaglandin E2 EP2 receptor reduces oxidative damage and amyloid burden in a model of Alzheimer's disease. J Neurosci. 2005;25(44):10180-10187.

23. Zhen G, et al. PGE2 EP1 receptor exacerbated neurotoxicity in a mouse model of cerebral ischemia and Alzheimer's disease. Neurobiol Aging. 2012;33(9):2215-2219.

24. Keene CD, et al. Suppressed accumulation of cerebral amyloid $\{$ beta $\}$ peptides in aged transgenic Alzheimer's disease mice by transplantation with wild-type or prostaglandin E2 receptor subtype 2-null bone marrow. Am J Pathol. 2010;177(1):346-354.

25. Shie FS, Montine KS, Breyer RM, Montine TJ. Microglial EP2 as a new target to increase amyloid beta phagocytosis and decrease amyloid beta-induced damage to neurons. Brain Pathol. 2005;15(2):134-138.

26. Johansson JU, et al. Suppression of inflammation with conditional deletion of the prostaglandin E2 EP2 receptor in macrophages and brain microglia. J Neurosci. 2013;33(40):16012-16032.

27. Mucke L, Selkoe DJ. Neurotoxicity of amyloid beta-protein: synaptic and network dysfunction. Cold Spring Harb Perspect Med. 2012;2(7):a006338.

28. Woodling NS, et al. Suppression of Alzheimerassociated inflammation by microglial prostaglandin-E2 EP4 receptor signaling. J Neurosci. 2014;34(17):5882-5894.

29. Shi J, Johansson J, Woodling NS, Wang Q, Montine TJ, Andreasson K. The prostaglandin E2
E-prostanoid 4 receptor exerts anti-inflammatory effects in brain innate immunity. JImmunol. 2010;184(12):7207-7218.

30. Savonenko A, et al. Episodic-like memory deficits in the APPswe/PS1dE9 mouse model of Alzheimer's disease: relationships to beta-amyloid deposition and neurotransmitter abnormalities. Neurobiol Dis. 2005;18(3):602-617.

31. Boillee $\mathrm{S}$, et al. Onset and progression in inherited ALS determined by motor neurons and microglia. Science. 2006;312(5778):1389-1392.

32. Morris GP, Clark IA, Zinn R, Vissel B. Microglia: a new frontier for synaptic plasticity, learning and memory, and neurodegenerative disease research. Neurobiol Learn Mem. 2013;105:40-53.

33. Figueiredo CP, et al. Memantine rescues transient cognitive impairment caused by high-molecular-weight abeta oligomers but not the persistent impairment induced by low-molecular-weight oligomers. J Neurosci. 2013;33(23):9626-9634.

34. Heneka MT, Landreth GE, Hull M. Drug insight: effects mediated by peroxisome proliferatoractivated receptor- $\gamma$ in CNS disorders. Nat Clin Pract Neurol. 2007;3(9):496-504.

35. Yamanaka M, Ishikawa T, Griep A, Axt D, Kummer MP, Heneka MT. PPAR $\gamma /$ RXR $\alpha$-induced and CD36-mediated microglial amyloid- $\beta$ phagocytosis results in cognitive improvement in amyloid precursor protein/presenilin 1 mice. J Neurosci. 2012;32(48):17321-17331.

36. Chawla A, et al. A PPAR $\gamma$-LXR-ABCA1 pathway in macrophages is involved in cholesterol efflux and atherogenesis. Mol Cell. 2001;7(1):161-171.

37. Laffitte BA, et al. LXRs control lipid-inducible expression of the apolipoprotein $\mathrm{E}$ gene in macrophages and adipocytes. Proc Natl Acad Sci U S A. 2001;98(2):507-512.

38. Jiang Q, et al. ApoE promotes the proteolytic degradation of A $\beta$. Neuron. 2008;58(5):681-693.

39. Mandrekar-Colucci S, Karlo JC, Landreth GE. Mechanisms underlying the rapid peroxisome proliferator-activated receptor- $\gamma$-mediated amyloid clearance and reversal of cognitive deficits in a murine model of Alzheimer's disease. JNeurosci. 2012;32(30):10117-10128.

40. Cramer PE, et al. ApoE-directed therapeutics rapidly clear $\beta$-amyloid and reverse deficits in $\mathrm{AD}$ mouse models. Science. 2012;335(6075):1503-1506.

41. Fitz NF, Cronican AA, Lefterov I, Koldamova R. Comment on "ApoE-directed therapeutics rapidly clear $\beta$-amyloid and reverse deficits in $\mathrm{AD}$ mouse models". Science. 2013;340(6135):924-c.

42. Han J, Sridevi P, Ramirez M, Ludwig KJ, Wang JY. $\beta$-Catenin-dependent lysosomal targeting of internalized tumor necrosis factor- $\alpha$ suppresses 
caspase- 8 activation in apoptosis-resistant colon cancer cells. Mol Biol Cell. 2013;24(4):465-473.

43. Fontana L, Partridge L, Longo VD. Extending healthy life span - from yeast to humans. Science. 2010;328(5976):321-326.

44. Fernandez AM, Torres-Aleman I. The many faces of insulin-like peptide signalling in the brain. Nat Rev Neurosci. 2012;13(4):225-239.

45. Ueno $\mathrm{M}$, et al. Layer $\mathrm{V}$ cortical neurons require microglial support for survival during postnatal development. Nat Neurosci. 2013;16(5):543-551.

46. Sze CI, Troncoso JC, Kawas C, Mouton P, Price DL, Martin LJ. Loss of the presynaptic vesicle protein synaptophysin in hippocampus correlates with cognitive decline in Alzheimer disease. J Neuropathol Exp Neurol. 1997;56(8):933-944.

47. Sanchez-Varo R, et al. Abnormal accumulation of autophagic vesicles correlates with axonal and synaptic pathology in young Alzheimer's mice hippocampus. Acta Neuropathol. 2012;123(1):53-70.

48. Shao CY, Mirra SS, Sait HB, Sacktor TC, Sigurdsson EM. Postsynaptic degeneration as revealed by PSD-95 reduction occurs after advanced $A \beta$ and tau pathology in transgenic mouse models of Alzheimer's disease. Acta Neuropathol. 2011;122(3):285-292.

49. Fiala M, et al. Ineffective phagocytosis of amyloid- $\beta$ by macrophages of Alzheimer's disease patients. J Alzheimers Dis. 2005;7(3):221-232.

50. Mawuenyega KG, et al. Decreased clearance of CNS $\beta$-amyloid in Alzheimer's disease. Science. 2010;330(6012):1774.

51. Adler AS, Sinha S, Kawahara TL, Zhang JY, Segal E, Chang HY. Motif module map reveals enforcement of aging by continual NF- $\mathrm{B}$ activity. Genes Dev. 2007;21(24):3244-3257.

52. Landreth GE, et al. Response to comments on "ApoE-directed therapeutics rapidly clear $\beta$-amyloid and reverse deficits in AD mouse models". Science. 2013;340(6135):924-g.

53. McNamara P, Seo SB, Rudic RD, Sehgal A, Chakravarti D, FitzGerald GA. Regulation of CLOCK and MOP4 by nuclear hormone receptors in the vasculature: a humoral mechanism to reset a peripheral clock. Cell. 2001;105(7):877-889.

54. Musiek ES, et al. Circadian clock proteins regulate neuronal redox homeostasis and neurodegeneration. J Clin Invest. 2013;123(12):5389-5400.

55. Kondratova AA, Kondratov RV. The circadian clock and pathology of the ageing brain. Nat Rev Neurosci. 2012;13(5):325-335.

56. Peek CB, et al. Circadian clock NAD+ cycle drives mitochondrial oxidative metabolism in mice. Science. 2013;342(6158):1243417.

57. Talbot K, et al. Demonstrated brain insulin resistance in Alzheimer's disease patients is associated with IGF-1 resistance, IRS-1 dysregulation, and cognitive decline. JClin Invest. 2012;122(4):1316-1338.

58. Carro E, Trejo JL, Gomez-Isla T, LeRoith D, Torres-Aleman I. Serum insulin-like growth factor I regulates brain amyloid- $\beta$ levels. Nat Med. 2002;8(12):1390-1397.

59. Dziennis S, et al. The CD11b promoter directs high-level expression of reporter genes in macrophages in transgenic mice. Blood. 1995;85(2):319-329.

60. Ferron M, Vacher J. Targeted expression of Cre recombinase in macrophages and osteoclasts in transgenic mice. Genesis. 2005;41(3):138-145.

61. Villeda SA, et al. The ageing systemic milieu negatively regulates neurogenesis and cognitive function. Nature. 2011;477(7362):90-94.

62 . Villeda SA, et al. Young blood reverses age-related impairments in cognitive function and synaptic plasticity in mice. Nat Med. 2014;20(6):659-663.

63. Jankowsky JL, Slunt HH, Ratovitski T, Jenkins NA, Copeland NG, Borchelt DR. Co- expression of multiple transgenes in mouse CNS: a comparison of strategies. Biomol Eng. 2001;17(6):157-165.

64. Breyer RM, Kennedy CR, Zhang Y, Guan Y, Breyer MD. Targeted gene disruption of the prostaglandin E2 EP2 receptor. Adv Exp Med Biol. 2002;507:321-326.

65. Kennedy CR, et al. Salt-sensitive hypertension and reduced fertility in mice lacking the prostaglandin EP2 receptor. Nat Med.1999;5(2):217-220.

66. Yang T, et al. Small molecule, non-peptide p75 ligands inhibit A $\beta$-induced neurodegeneration and synaptic impairment. PLoS One. 2008;3(11):e3604.

67. El Khoury J, et al. Ccr2 deficiency impairs microglial accumulation and accelerates progression of Alzheimer-like disease. Nat Med. 2007;13(4):432-438.

68. El Khoury JB, et al. CD36 mediates the innate host response to beta-amyloid. J Exp Med. 2003;197(12):1657-1666.

69. Piermartiri TC, et al. Atorvastatin prevents hippocampal cell death, neuroinflammation and oxidative stress following amyloid- $\beta(1-40)$ administration in mice: evidence for dissociation between cognitive deficits and neuronal damage. Exp Neurol. 2010;226(2):274-284.

70. Melnikova T, et al. Cycloxygenase-2 activity promotes cognitive deficits but not increased amyloid burden in a model of Alzheimer's disease in a sex-dimorphic pattern. Neuroscience. 2006;141(3):1149-1162.

71. Dere E, Huston JP, De Souza Silva MA. The pharmacology, neuroanatomy and neurogenetics of one-trial object recognition in rodents. Neurosci Biobehav Rev. 2007;31(5):673-704.

72. Clelland CD, et al. A functional role for adult hippocampal neurogenesis in spatial pattern separation. Science. 2009;325(5937):210-213. 